\title{
Frobenius monads and pseudomonoids
}

Ross Street

April 2004

\begin{abstract}
Six equivalent definitions of Frobenius algebra in a monoidal category are provided. In a monoidal bicategory, a pseudoalgebra is Frobenius if and only if it is star autonomous. Autonomous pseudoalgebras are also Frobenius. What it means for a morphism of a bicategory to be a projective equivalence is defined; this concept is related to "strongly separable" Frobenius algebras and "weak monoidal Morita equivalence". Wreath products of Frobenius algebras are discussed.
\end{abstract}

\section{Introduction}

Over the last two decades, the relevance of categories to physics has become widely acknowledged in at least two particular areas: quantum group theory QGT (see [JS3], [Kas], [Maj]) and topological quantum field theory TQFT (see [Ko], [KL]). Quantum groups arise from the Yang-Baxter equation of statistical mechanics, while each quantum group has a monoidal (or "tensor") category of representations. A two-dimensional TQFT can be regarded as a tensor-preserving functor from a monoidal category of 2-cobordisms to the category of vector spaces. Monoidal categories can be used to construct the known three-dimensional TQFTs while some four-dimensional TQFTs can be constructed using monoidal bicategories (see [Bz]). Both QGT and TQFT feature categories whose morphisms come from lowdimensional topology (braids, links, tangles, surfaces, and so on). Mainly for the benefit of readers from mathematical physics, in this introduction and the next section, we will warm up to the categorical notions just mentioned and a few others we require.

Of course, the use in physics of (classical) groups and their representations goes back many score years. A lot of information about a group $G$ is contained in its characters. Characters are group morphisms from $G$ into the multiplicative monoid of an appropriate field $k$. In other words, we find a category (in this case the category of monoids) where G and $\mathrm{k}$ both live as objects so that it makes sense to look at morphisms between these objects.

Representations reveal even more about $G$ than characters yet can be introduced using the same philosophy. The group $G$ can be regarded as a category $\Sigma G$ having only one object and every morphism invertible. Although this could be taken as the definition of group, it is often helpful to maintain a notational distinction between the group and the one-object category (after all, groups can be defined in alternative categorical terms as discrete closed monoidal categories). We think of $\Sigma G$ as a kind of suspension of $G$ where the morphisms of $\Sigma G$ are the elements of G. Since we have put $G$ into the category Cat of categories, we can look at morphisms from $\Sigma G$ into other categories such as the category Vect ${ }_{k}$ of vector spaces over $k$. These are precisely linear representations of the group G: a morphism of categories $\mathrm{F}: \Sigma \mathrm{G} \longrightarrow$ Vect $_{\mathrm{k}}$ (functor) takes the one object of $\Sigma \mathrm{G}$ to the vector space underlying the representation and the morphisms of $\Sigma G$ to action by those group elements. 
This paper is concerned with the identification of mathematical structure on objects of interest. The structure of particular interest is an abstraction of Frobenius algebra; we will soon recall the basic concept. The connection between TQFT and Frobenius algebras is pointed out in $[\mathrm{Ko}]$ and we proceed to outline how that works. Some connection between quantum groups and Frobenius algebras is already apparent from the fact that quantum groups are Hopf algebras and finite-dimensional Hopf algebras are Frobenius [LSw]. We intend to deepen the connection between Frobenius algebras and quantum group theory.

A k-algebra A is called Frobenius when it is equipped with an exact pairing

$$
\sigma: \mathrm{A} \otimes \mathrm{A} \longrightarrow \mathrm{k}
$$

satisfying the condition

$$
\sigma((a b) \otimes c)=\sigma(a \otimes(b c))
$$

for all elements $\mathrm{a}, \mathrm{b}$ and $\mathrm{c}$ of $\mathrm{A}$. (The exact bilinear pairing in the sense of monoidal category theory here means that $\mathrm{A}$ is finite dimensional as a vector space and the pairing provides an isomorphism between $A$ and its linear dual.) In fact, $\sigma$ is determined by a linear function $\varepsilon: A \longrightarrow k$ via the formulas $\varepsilon(a)=\sigma(1, a)$ and $\sigma(a, b)=\varepsilon(a b)$. The group algebra $\mathrm{kG}$ of any finite group $\mathrm{G}$ is Frobenius when equipped with the form $\sigma$ that has $\sigma(\mathrm{x}, \mathrm{y})=1$ if and only if $\mathrm{xy}=1$.

We shall recall in Section 1 how each Frobenius algebra becomes a coalgebra. However, in the case where the Frobenius algebra comes from a finite-dimensional Hopf algebra, this coalgebra is not the same as the coalgebra underlying the Hopf algebra. For one thing, a morphism of Frobenius algebras (preserving the algebra and coalgebra structure) is invertible (see [Ko; Section 2.4]) whereas Hopf algebra morphisms between group algebras are in bijection with group morphisms.

Each commutative Frobenius algebra determines (uniquely up to isomorphism) a 2dimensional TQFT; that is, a tensor-preserving functor from the monoidal category 2-Cob of 2-dimensional cobordisms to Vect $_{k}$. More precisely, the category of commutative Frobenius $\mathrm{k}$-algebras is equivalent to the category of symmetric strong-monoidal functors from 2-Cob to $\operatorname{Vect}_{\mathrm{k}}$ (see [Ko; Theorem 3.3.2]). Both of these categories are actually groupoids: every morphism is invertible. We already mentioned this fact for Frobenius algebras, while the monoidal category 2-Cob is autonomous (that is, its objects all have both left and right duals) and this already implies that every morphism between 2D TQFTs is invertible.

A category $\mathcal{V}$ is monoidal when it is equipped with a functor $\otimes: \mathcal{V} \times \mathcal{V} \longrightarrow \mathcal{V}$ (called the tensor product), an object I of $\mathcal{V}$ (called the tensor unit), and three natural families of isomorphisms

$$
(A \otimes B) \otimes C \cong A \otimes(B \otimes C), \quad I \otimes A \cong A \cong A \otimes I
$$

in $\mathcal{V}$ (called associativity and unital constraints), such that the pentagon, involving the five ways of bracketing four objects, commutes, and the associativity constraint with $\mathrm{B}=\mathrm{I}$ is compatible with the unit constraints. Call $\mathcal{V}$ braided when it is equipped with a natural family of 
isomorphisms

$$
\mathrm{c}_{\mathrm{A}, \mathrm{B}}: \mathrm{A} \otimes \mathrm{B} \cong \mathrm{B} \otimes \mathrm{A}
$$

(called the braiding) satisfying two conditions (one expressing $\mathrm{c}_{\mathrm{A} \otimes \mathrm{B}, \mathrm{C}}$ in terms of associativity constraints, $1_{A} \otimes c_{B, C}$ and $C_{C, A} \otimes 1_{B}$, and a similar one for $c_{A, B \otimes C}$. A braiding is a symmetry when $\mathrm{C}_{\mathrm{B}, \mathrm{A}} \circ \mathrm{C}_{\mathrm{A}, \mathrm{B}}=1_{\mathrm{A} \otimes \mathrm{B}}$. A monoidal category is called strict when the associativity and unital constraints are identities.

Monoids can be defined in any monoidal category $\mathcal{V}$. A monoid in $\mathcal{V}$ is an object A equipped with a multiplication $\mu: \mathrm{A} \otimes \mathrm{A} \longrightarrow \mathrm{A}$ and a unit $\eta: \mathrm{I} \longrightarrow \mathrm{A}$ satisfying unital and associativity conditions. A monoid in the category Set of sets, where the tensor product is cartesian product, is a monoid in the usual sense. If we use the coproduct (disjoint union) in Set as tensor product, every set has a unique monoid structure. A monoid in Vect $_{k}$, with the usual tensor product of vector spaces, is precisely a k-algebra; monoids in monoidal k-linear categories are also sometimes called algebras. A monoid in the category Cat of categories (where the morphisms are functors and the tensor product is cartesian product) is a strict monoidal category. For any category $\mathcal{A}$, the category $[\mathcal{A}, \mathcal{A}]$ of endofunctors on $\mathcal{A}$ becomes strict monoidal by taking composition as the tensor product: a monoid in $[\mathcal{A}, \mathcal{A}]$ is called a monad on $\mathcal{A}$ (see [ML] for the theory of monads and their algebras).

Frobenius structure on a monoid makes sense in any monoidal category. We recall this in Section 1 where we assemble some facts about Frobenius monoids. Many of the facts are scattered throughout the literature. To begin with, for a concrete case that denies us the luxury of symmetry, we express the results in terms of monads on categories; but clearly the results carry over to monoids in general monoidal categories.

In a symmetric (or even braided) monoidal category we can define commutative monoids. In fact, what is shown in [Ko] is that $2-\mathrm{Cob}$ possesses a distinguished commutative Frobenius monoid and that every commutative Frobenius monoid in every symmetric monoidal category is the image, under an essentially unique symmetric monoidal functor, of the distinguished one.

Notice from the above discussion that it is the strict monoidal categories, and not the general ones, that are genuinely examples of monoids in Cat. The reason non-strictness arises is that Cat is a 2-category: natural transformations between the functors provide Cat with the 2-dimensional structure of 2-morphisms or 2-cells and their compositions. Cartesian product is compatible with the 2-cells and so Cat is actually a monoidal 2-category.

Composition of functors is strictly associative so Cat itself is stricter than it might be in the 2-dimensional setting. This leads to a weaker version of 2-category due to Bénabou [Bu]. A bicategory $\mathcal{B}$ has objects, and, for objects $\mathrm{A}$ and $\mathrm{B}$, we have a category $\mathcal{B}(\mathrm{A}, \mathrm{B}$ ) (called a homcategory) whose objects are called morphisms $\mathrm{f}: \mathrm{A} \longrightarrow \mathrm{B}$ of $\mathcal{B}$, whose morphisms are called 2cells $\theta: \mathrm{f} \Rightarrow \mathrm{g}: \mathrm{A} \longrightarrow \mathrm{B}$ of $\mathcal{B}$, and whose composition is called vertical composition in $\mathcal{B}$; there are functors 


$$
-\circ-: \mathcal{B}(\mathrm{B}, \mathrm{C}) \times \mathcal{B}(\mathrm{A}, \mathrm{B}) \longrightarrow \mathcal{B}(\mathrm{A}, \mathrm{C})
$$

(called horizontal composition) and morphisms $1_{\mathrm{A}}: \mathrm{A} \longrightarrow \mathrm{A} \quad$ (called identity morphisms). Horizontal composition is associative with units the identity morphisms: but only up to invertible 2-cells that are just like the associativity and unital constraints of a monoidal category. In fact, for each object $\mathrm{A}$, the category $\mathcal{B}(\mathrm{A}, \mathrm{A})$ becomes monoidal by using the horizontal composition as tensor product; this is the 2-dimensional version of the fact that endomorphism sets in any category are monoids. An object A together with a monoid $t, \eta$, $\mu$ in the monoidal category $\mathcal{B}(\mathrm{A}, \mathrm{A})$ is called a monad in $\mathcal{B}$. Each monoidal category $\mathcal{V}$ can be regarded as a bicategory $\Sigma \mathcal{V}$ with one object; the endohom of that object is $\mathcal{V}$ and the horizontal composition is the tensor product of $\mathcal{V}$.

In a bicategory, Bénabou introduced the operation of pasting which is applied to diagrams such as

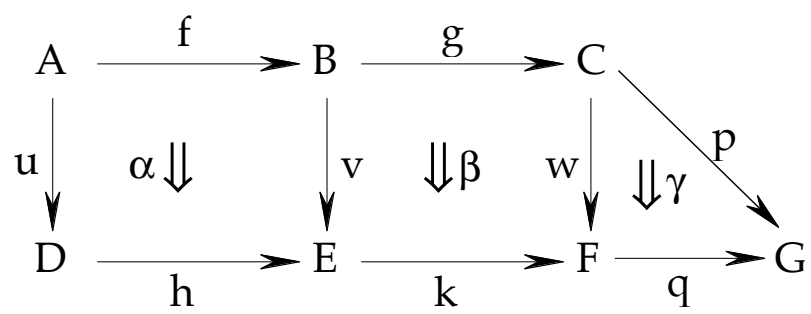

in which the left square depicts a 2-cell $\alpha: \mathrm{v} \circ \mathrm{f} \Rightarrow \mathrm{h} \circ \mathrm{u}$, the middle square depicts a 2-cell $\beta: \mathrm{w} \circ \mathrm{g} \Rightarrow \mathrm{k} \circ \mathrm{v}$, and the right triangle depicts a 2-cell $\gamma: \mathrm{p} \Rightarrow \mathrm{q} \circ \mathrm{w}$. Once a bracketing is chosen for the upper path $\mathrm{p} \circ \mathrm{g} \circ \mathrm{f}$ yielding a morphism $\mathrm{s}: \mathrm{A} \longrightarrow \mathrm{G}$ and a bracketing is chosen for the lower path $\mathrm{q} \circ \mathrm{k} \circ \mathrm{h} \circ \mathrm{u}$ yielding a morphism $\mathrm{t}: \mathrm{A} \longrightarrow \mathrm{G}$, there is a unique 2cell $\sigma: \mathrm{s} \Rightarrow \mathrm{t}$ determined by the diagram using the compositions and constraints of the bicategory; we call $\sigma$ the pasting composite of the diagram. In the exemplary diagram, taking $\mathrm{s}=\mathrm{p} \circ(\mathrm{g} \circ \mathrm{f})$ and $\mathrm{t}=\mathrm{q} \circ(\mathrm{k} \circ(\mathrm{h} \circ \mathrm{u}))$, we obtain $\sigma$ as the composite

$$
\begin{gathered}
\mathrm{p} \circ(\mathrm{g} \circ \mathrm{f}) \stackrel{\alpha \circ(\mathrm{g} \circ \mathrm{f})}{\longrightarrow}(\mathrm{q} \circ \mathrm{w}) \circ(\mathrm{g} \circ \mathrm{f}) \cong \mathrm{q} \circ((\mathrm{w} \circ \mathrm{g}) \circ \mathrm{f}) \stackrel{\mathrm{q} \circ(\beta \circ f)}{\longrightarrow} \\
\mathrm{q} \circ((\mathrm{k} \circ \mathrm{v}) \circ \mathrm{f}) \cong \mathrm{q} \circ(\mathrm{k} \circ(\mathrm{v} \circ \mathrm{f})) \stackrel{\mathrm{q} \circ(\mathrm{k} \circ \alpha)}{\longrightarrow} \mathrm{q} \circ(\mathrm{k} \circ(\mathrm{h} \circ \mathrm{u}))
\end{gathered}
$$

in the category $\mathcal{B}(\mathrm{A}, \mathrm{G})$. For simplicity we sometimes ignore the brackets and constraints in describing such composites (that is, we act as if we were in a 2-category). String diagrams can also be used: suffice it to say here that the string diagram corresponding to our pasting diagram is as follows where the 2-cells label vertices, the morphisms label edges, and the objects label faces. 


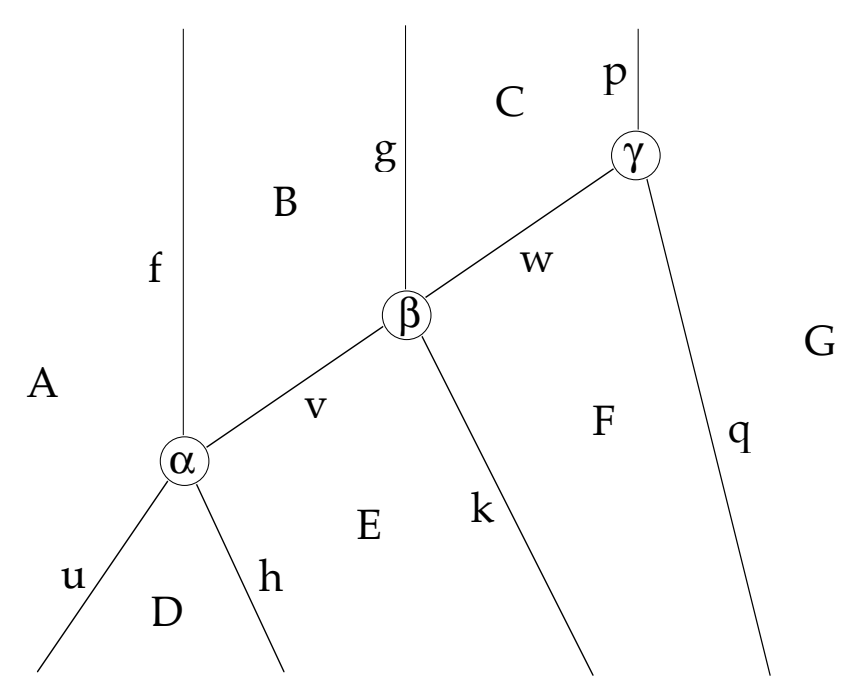

Each bicategory $\mathcal{B}$ has duals $\mathcal{B}^{\text {op }}, \mathcal{B}^{\text {co }}$ and $\mathcal{B}^{\text {coop }}$ : the first has morphisms reversed, the second has 2-cells reversed, while the third has both morphisms and 2-cells reversed.

In any bicategory $\mathcal{B}$, we can define adjoint morphisms: we say $\mathrm{u}: \mathrm{A} \longrightarrow \mathrm{B}$ is right adjoint to $\mathrm{f}: \mathrm{B} \longrightarrow \mathrm{A}$ when there are 2-cells

$$
\varepsilon: \mathrm{f} \circ \mathrm{u} \Rightarrow 1_{\mathrm{A}}: \mathrm{A} \longrightarrow \mathrm{A} \quad \text { and } \quad \eta: 1_{\mathrm{B}} \Rightarrow \mathrm{u} \circ \mathrm{f}: \mathrm{B} \longrightarrow \mathrm{B}
$$

(called the counit and unit) satisfying the following 2-cell equations: $(\varepsilon \circ f)(f \circ \eta)=1_{f} \quad$ and $(\mathrm{u} \circ \varepsilon)(\eta \circ \mathrm{u})=1_{\mathrm{u}}$ (the reader might like to draw the pasting and string diagrams as an exercise). We say $\mathrm{u}$ is an equivalence when it is right adjoint to an $\mathrm{f}$ with invertible counit and unit (actually, in this case we do not need to have the 2-cell equations; the unit can always be rechosen so that they hold). If $u$ is right adjoint to $f$ then $B$ together with $t=u \circ f$ form $a$ monad in $\mathcal{B}$ with unit $\eta$ and multiplication $\mathrm{u} \circ \varepsilon \circ \mathrm{f}$.

For any morphism $\mathrm{f}: \mathrm{X} \longrightarrow \mathrm{Y}$ in the bicategory, we write $\mathrm{f}^{*}: \mathrm{Y} \longrightarrow \mathrm{X}$ for a right adjoint should there be one.

An object $\mathrm{U}$ of a monoidal category $\mathcal{V}$ is said to be right dual to an object $\mathrm{V}$ when the morphism $\mathrm{U}$ is right adjoint to $\mathrm{V}$ in the bicategory $\Sigma \mathcal{V}$. For example, a vector space $\mathrm{U}$ is a right dual in Vect $\mathrm{k}_{\mathrm{k}}$ if and only if $\mathrm{U}$ is finite dimensional; in this case, $U$ is right dual to the space $\operatorname{Vect}_{\mathrm{k}}(\mathrm{U}, \mathrm{k})$ of linear functions from $\mathrm{U}$ to $\mathrm{k}$. A monoidal category is called right autonomous when every object has a right dual; it is called autonomous when every object has both a left and right dual. If $\mathcal{V}$ is symmetric then every right dual is also a left dual.

There is a weaker kind of monoidal duality that was conceived by Barr (see [Ba1], [Ba2], [Ba3]) based on examples in topological algebra yet the notion has received a lot of attention by computer scientists interested in Girard's "linear logic". A monoidal category $\mathcal{V}$ is said to be $*$-autonomous when there is an equivalence of categories $\mathrm{S}: \mathcal{V} \longrightarrow \mathcal{V}^{\text {op }}$ and a natural family of isomorphisms 


$$
\mathcal{V}(\mathrm{U} \otimes \mathrm{V}, \mathrm{I}) \cong \mathcal{V}(\mathrm{V}, \mathrm{SU})
$$

Each autonomous monoidal $\mathcal{V}$ is *-autonomous with $\mathrm{SU}$ a right dual for $\mathrm{U}$; the existence of left duals is needed for this $S$ to be an equivalence.

If $\mathcal{B}$ and $\mathcal{D}$ are bicategories, a lax functor (or "morphism") $\mathrm{F}: \mathcal{B} \longrightarrow \mathcal{D}$ consists of a function between the sets of objects together with functors

$$
\mathrm{F}_{\mathrm{A}, \mathrm{B}}: \mathcal{B}(\mathrm{A}, \mathrm{B}) \longrightarrow \mathcal{D}(\mathrm{FA}, \mathrm{FB})
$$

and invertible 2-cells $\mathrm{F}_{\mathrm{B}, \mathrm{C}}(\mathrm{g}) \circ \mathrm{F}_{\mathrm{A}, \mathrm{B}}(\mathrm{f}) \Rightarrow \mathrm{F}_{\mathrm{A}, \mathrm{C}}(\mathrm{g} \circ \mathrm{f})$, natural in $\mathrm{f}$ and $\mathrm{g}$, and $1_{\mathrm{FA}} \Rightarrow \mathrm{F}\left(1_{\mathrm{A}}\right)$; these 2-cells satisfy three conditions very much like associativity and unital conditions for a monoid. When these structural 2-cells are all invertible the lax functor is called a pseudofunctor (or "homomorphism"). A biequivalence is a pseudofunctor $F$ for which each $\mathrm{F}_{\mathrm{A}, \mathrm{B}}$ is an equivalence of categories and, for each object $\mathrm{D}$ of $\mathcal{D}$, there is an object $\mathrm{A}$ of $\mathcal{B}$ and an

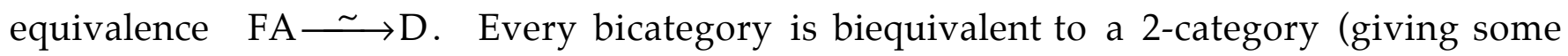
justification for leaving out constraints when writing equations).

Between pseudofunctors there are pseudonatural transformations: these are a 2-dimensional version of natural transformation in which the naturality equations are "broken" by asking them to hold only up to extra invertible 2-cells that satisfy some further conditions (see [KS] for example). There is a bicategory $\operatorname{Hom}(\mathcal{B}, \mathcal{D})$ whose objects are the pseudofunctors from $\mathcal{B}$ to $\mathcal{D}$, whose morphisms are the pseudonatural transformations, and whose 2-cells are called modifications. We shall only have need of modifications in one place in this paper (in defining "scalars") and there we shall spell out exactly what we mean.

A 2-category can be defined to be a strict bicategory: one in which the associativity and unital constraints are identities. So Cat is special among bicategories; it is strict. Cartesian product is also special among monoidal structures on bicategories; it is stricter in many ways than required of a general monoidal bicategory. A bicategory $\mathcal{B}$ is monoidal when it is equipped with a pseudofunctor $\otimes: \mathcal{B} \times \mathcal{B} \longrightarrow \mathcal{B}$ and an object I together with associativity and unital constraints much like a monoidal category except that they need only be equivalences rather than isomorphisms and they need only satisfy the conditions up to further selected isomorphisms that themselves satisfy conditions. Monoidal bicategories are not all monoidally biequivalent to monoidal 2-categories but some degree of strictness can be attained. We do not need more detail than this; however, the interested reader can consult [DS1] and [McC].

In any monoidal bicategory it is possible to define pseudomonoids; these are like monoids except that the associativity and unital conditions only hold up to invertible 2-cells that are called associativity and unit constraints; they are required to satisfy conditions that are said to express coherence; again, a reference is [DS1]. In particular, a pseudomonoid in Cat is precisely a monoidal category. Hence a pseudomonoid is also called a "monoidal object" of the monoidal bicategory. 
In Section 2 we continue this review of categorical structures highlighting enriched categories.

In Section 3 we define what it means for a pseudomonoid in any monoidal bicategory to be Frobenius. It is an easy corollary of results of [DMS] that every autonomous pseudomonoid (whose unit has a right adjoint) is Frobenius. As we have mentioned, finite dimensional Hopf algebras are known to be Frobenius, yet our corollary provides a setting in which even the more general quasi-Hopf algebras of Drinfeld are Frobenius irrespective of dimension. Another example is any autonomous monoidal $\mathcal{V}$-category. In [DS2], we showed how quantum groups (and more generally "quantum groupoids") and star-autonomous monoidal categories are instances of the same mathematical structure. Although the term Frobenius was not used in [DS2], the star-autonomy defined there is precisely the higher-dimensional version of Frobenius structure.

Section 4 is largely inspired by the discussion of "weak monoidal Morita equivalence" in [Mü1] and [Mü2] where it is shown that monoidal categories that are equivalent in this weak sense still give rise to the same state sum invariants of closed oriented 3-manifolds (see [BW1] and [BW2]). We define a notion of projective equivalence between objects in any bicategory. In the same general setting, we define what it means for a Frobenius monad to be strongly separable and relate this to projective equivalence. Both concepts require the abstract notion of "scalar" determined by the bicategory. In the bicategory of k-linear categories for a commutative ring $\mathrm{k}$, the scalars are in bijection with elements of $\mathrm{k}$. Section 5 says a little about Morita equivalence.

Finally, in Section 6, we discuss wreath products of Frobenius algebras. This is done at the level of generalized distributive laws between monads as developed in [LSt].

\section{§1. Frobenius monads}

Let $\mathbf{T}=(\mathrm{T}, \eta, \mu)$ be a monad on a category $X$. We write $X^{\mathrm{T}}$ for the category of $\mathbf{T}$ algebras in the sense of [EM] (although those authors called monads "triples"). We write $\mathrm{U}^{\mathrm{T}}: X^{\mathrm{T}} \longrightarrow X$ for the forgetful functor and $\mathrm{F}^{\mathrm{T}}: X \longrightarrow X^{\mathrm{T}}$ for its left adjoint. Similarly, for a comonad $\mathrm{G}=(\mathrm{G}, \varepsilon, \delta)$, we write $X^{\mathrm{G}}$ for the category of $\mathrm{G}$-coalgebras, we write $\mathrm{V}^{\mathrm{G}}: X^{\mathrm{G}} \longrightarrow X$ for the forgetful functor, and we write $\mathrm{C}^{\mathrm{G}}: X \longrightarrow X^{\mathrm{G}}$ for the right adjoint of $\mathrm{V}^{\mathrm{G}}$.

Before defining Frobenius monads and finding several equivalent definitions, we shall recall the results in Section 3 of $[E M]$ on adjoint monads. Let $\mathbf{T}=(T, \eta, \mu)$ be a monad on a category $X$ such that the endofunctor $\mathrm{T}$ has a right adjoint: our notation is $\mathrm{T} \dashv \mathrm{G}$ with counit $\sigma: \mathrm{TG} \longrightarrow 1$ and unit $\rho: 1 \longrightarrow \mathrm{GT}$. Eilenberg and Moore showed that:

AM1. $G=(G, \varepsilon, \delta)$ is a comonad where $\varepsilon$ and $\delta$ are the mates (in the sense of [KS]) of $\eta$ and $\mu$ under adjunction, with the explicit formulas being 


$$
\varepsilon=\sigma \circ \eta \mathrm{G} \text { and } \delta=\mathrm{G}^{2} \sigma \circ \mathrm{G}^{2} \mu \mathrm{G} \circ \mathrm{G} \rho \mathrm{TG} \circ \rho \mathrm{G},
$$

and the comonad $\mathbf{G}$ is said to be right adjoint to the $\operatorname{monad} \mathbf{T}$;

AM2. mateship under adjunction of action and coaction defines an isomorphism of categories $X^{\mathrm{T}} \cong X^{\mathrm{G}}$ that commutes with the forgetful functors $\mathrm{U}^{\mathrm{T}}$ and $\mathrm{V}^{\mathrm{G}}$ into $X$;

AM3. each of the forgetful functors $\mathrm{U}^{\mathrm{T}}$ and $\mathrm{V}^{\mathrm{G}}$ has both left and right adjoints; and,

AM4. if $F \dashv U \dashv C$ then the comonad generated by $U \dashv C$ is right adjoint to the monad generated by $\mathrm{F} \dashv \mathrm{U}$.

We can add the following extra observation on adjoint monads; it is a trivial consequence of Beck's monadicity theorem [Bec]).

AM5. if $\mathrm{F} \dashv \mathrm{U} \dashv \mathrm{C}$ and $\mathrm{U}$ is conservative (that is, reflects invertibility of morphisms) then the comparison functor, into the category of Eilenberg-Moore algebras for the monad generated by $\mathrm{F} \dashv \mathrm{U}$, is an equivalence;

If $\mathbf{T}=(\mathrm{T}, \eta, \mu)$ is a monad on a category $X$ such that the endofunctor $\mathrm{T}$ has a left adjoint $\mathrm{H}$, we can apply the duality explained in [St1] to obtain five corresponding results. In particular, there is a comonad $\mathbf{H}=(\mathrm{H}, \varepsilon, \delta)$ for which there is an isomorphism $\chi_{\mathbf{T}} \cong X_{\mathbf{H}}$ where $\mathcal{X}_{\mathbf{T}}$ and $\mathcal{X}_{\mathbf{H}}$ are the Kleisli categories of the monad $\mathbf{T}$ and the comonad $\mathbf{H}$ respectively. Also, if a functor $\mathrm{F}: X \longrightarrow \mathcal{K}$ has a left adjoint, a right adjoint, and is essentially surjective on objects, then $\mathcal{K}$ is equivalent to the Kleisli categories for the appropriately generated monad and comonad on $X$.

Definition 1.1 A monad $\mathbf{T}=(\mathrm{T}, \eta, \mu)$ is called Frobenius when it is equipped with a natural transformation $\varepsilon: \mathrm{T} \longrightarrow 1$ such that there exists a natural transformation $\rho: 1 \longrightarrow \mathrm{T}^{2}$ satisfying the equations

$$
\mathrm{T} \mu \circ \rho \mathrm{T}=\mu \mathrm{T} \circ \mathrm{T} \rho \quad \text { and } \quad \mathrm{T} \varepsilon \circ \rho=\eta=\varepsilon \mathrm{T} \circ \rho .
$$

Lemma 1.2 For a Frobenius monad $\mathrm{T}$, put $\delta:=\mathrm{T} \mu \circ \rho \mathrm{T}=\mu \mathrm{T} \circ \mathrm{T} \rho$. Then
(a) $\mathrm{T} \mu \circ \delta \mathrm{T}=\delta \circ \mu=\mu \mathrm{T} \circ \mathrm{T} \delta$
(b) $\mathrm{T} \varepsilon \circ \delta=1_{\mathrm{T}}=\varepsilon \mathrm{T} \circ \delta$
(c) $\rho=\delta \circ \eta$.

Proof (a) $\mathrm{T} \mu \circ \delta \mathrm{T}=\mathrm{T} \mu \circ \mathrm{T} \mu \mathrm{T} \circ \rho \mathrm{T}^{2}=\mathrm{T} \mu \circ \mathrm{T}^{2} \mu \circ \rho \mathrm{T}^{2}=\mathrm{T} \mu \circ \rho \mathrm{T} \circ \mu=\delta \circ \mu$

(b) $\mathrm{T} \varepsilon \circ \delta=\mathrm{T} \varepsilon \circ \mu \mathrm{T} \circ \mathrm{T} \rho=\mu \circ \mathrm{T}^{2} \varepsilon \circ \mathrm{T} \rho=\mu \circ \mathrm{T} \eta=1_{\mathrm{T}}$

$$
\mu \mathrm{T} \circ \mathrm{T} \delta=\mu \mathrm{T} \circ \mathrm{T} \mu \mathrm{T} \circ \mathrm{T}^{2} \rho=\mu \mathrm{T} \circ \mu \mathrm{T}^{2} \circ \mathrm{T}^{2} \rho=\mu \mathrm{T} \circ \mathrm{T} \rho \circ \mu=\delta \circ \mu
$$

$$
\varepsilon \mathrm{T} \circ \delta=\varepsilon \mathrm{T} \circ \mathrm{T} \mu \circ \rho \mathrm{T}=\mu \circ \varepsilon \mathrm{T}^{2} \circ \rho \mathrm{T}=\mu \circ \eta \mathrm{T}=1_{\mathrm{T}}
$$

(c) $\delta \circ \eta=\mathrm{T} \mu \circ \rho \mathrm{T} \circ \eta=\mathrm{T} \mu \circ \mathrm{T}^{2} \eta \circ \rho=\rho$. QED 
Remark Condition (a) of Lemma 1.2 has occurred in the work of Carboni and Walters (see $[\mathrm{CW}]$ and [Cbn]) and of Boyer and Joyal (unfortunately [BJ] is unpublished but see [St4] for some details). The condition relates to separability of algebras and discreteness. Condition (b) expesses that $\varepsilon$ is a counit for the comultiplication $\delta$. Condition (c) suggests dually introducing $\sigma=\varepsilon \circ \mu$ as we shall now do.

Lemma 1.3 For a Frobenius monad $\mathrm{T}$, there is an adjunction $\mathrm{T} \rightarrow \mathrm{T}$ with counit $\sigma=\varepsilon \circ \mu: \mathrm{T}^{2} \longrightarrow 1$ and unit $\rho: 1 \longrightarrow \mathrm{T}^{2}$. Moreover, $\mathrm{G}=(\mathrm{T}, \varepsilon, \delta)$ is a right adjoint comonad for the monad $\mathbf{T}$.

Proof We shall do this using the string calculus (as justified by [JS1]). We use Lemma 1.2.

One of the counit/unitidentities is proved by the following calculation;look in a mirror for the proof of the other.
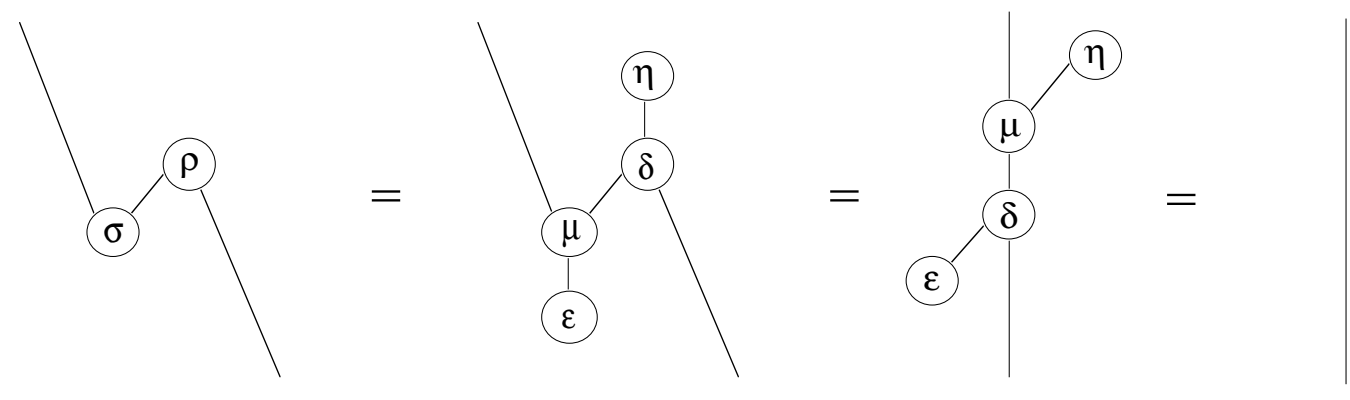

For the second sentence we need to show that $\delta=\mathrm{T}^{2} \sigma \circ \mathrm{T}^{2} \mu \mathrm{G} \circ \mathrm{T} \rho \mathrm{T}^{2} \circ \rho \mathrm{T}$ and $\varepsilon=\sigma \circ \eta \mathrm{T}$. The latter is easy since $\sigma \circ \eta \mathrm{T}=\varepsilon \circ \mu \circ \eta \mathrm{T}=\varepsilon$. For the former we have:
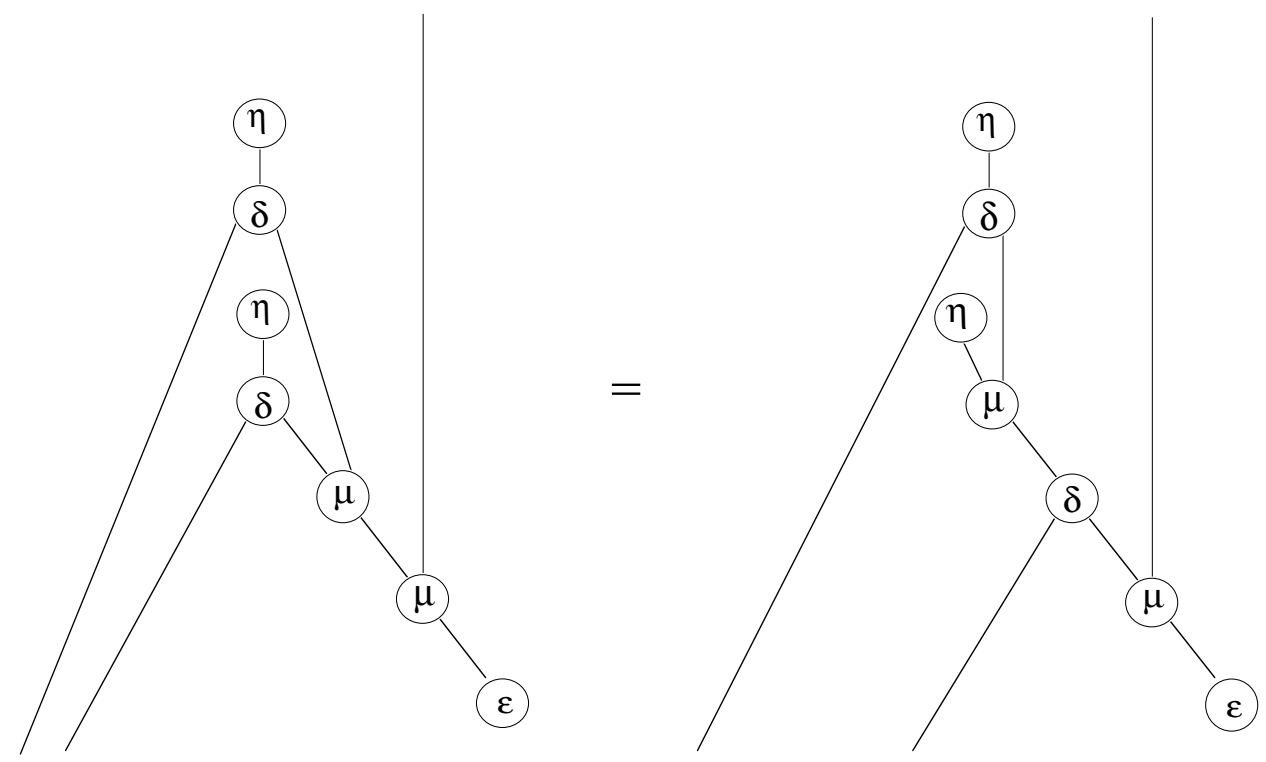

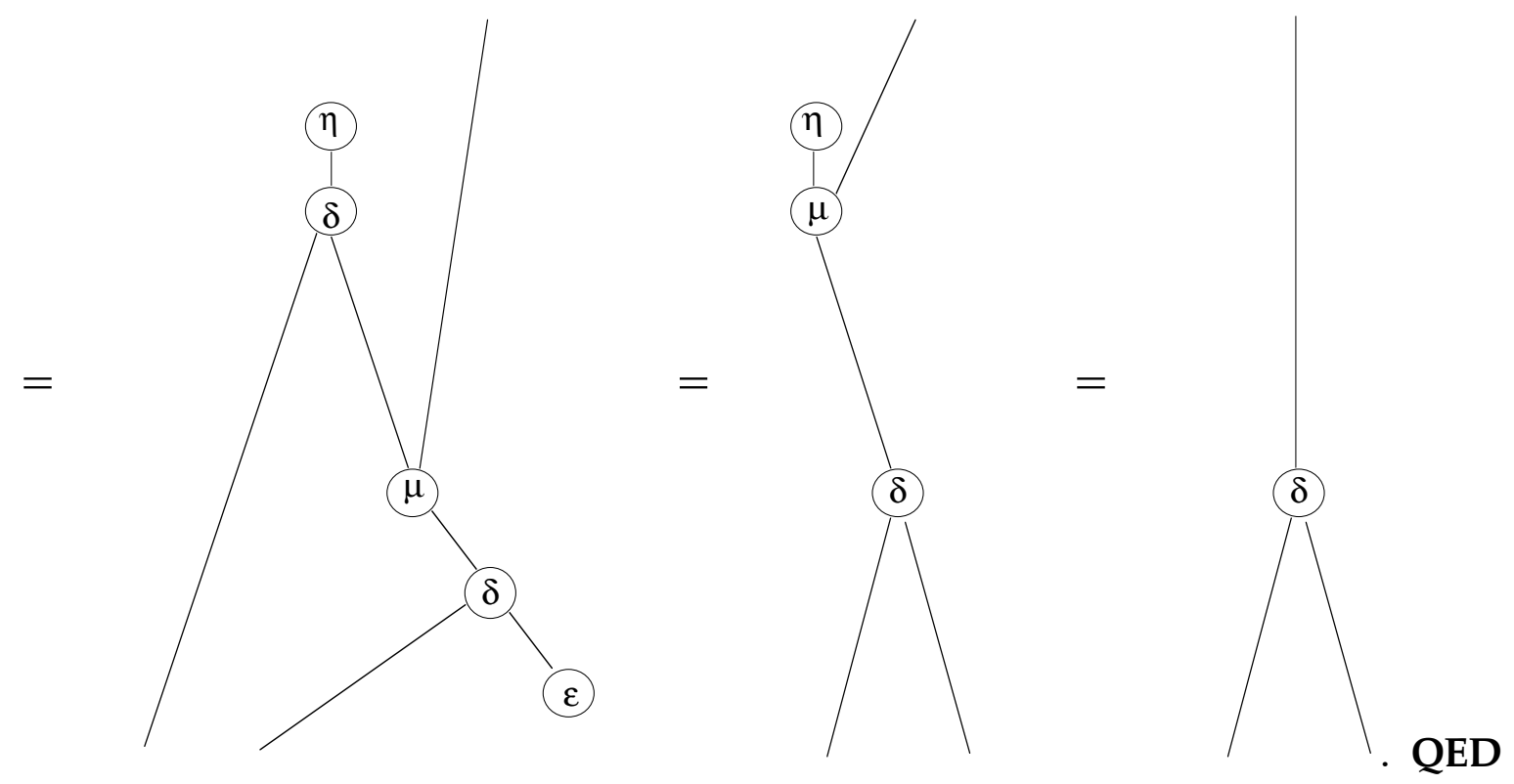

Remark (a) It follows from the first sentence of Lemma 1.3 that $\rho$ is uniquely determined by the monad $\mathbf{T}=(\mathrm{T}, \eta, \mu)$ and $\varepsilon$. This is because the counit $\sigma$ is determined by $\mu$ and $\varepsilon$, and the counit of any adjunction uniquely determines the unit.

(b) It is implicit (using AM1) in the second sentence of Lemma 1.3 that $\delta$ is coassociative.

Proposition 1.4 For a Frobenius monad $\mathbf{T}$, the left adjoint $\mathrm{F}^{\mathbf{T}}: X \longrightarrow X^{\mathbf{T}}$ to the forgetful functor $\mathrm{U}^{\mathrm{T}}: X^{\mathrm{T}} \longrightarrow X$ is also a right adjoint to $\mathrm{U}^{\mathbf{T}}$ with counit $\varepsilon$.

Proof By AM2 we know that there is an isomorphism of categories $\mathrm{K}: X^{\mathrm{T}} \cong X^{\mathrm{G}}$ such that $\mathrm{V}^{\mathrm{G}} \mathrm{K}=\mathrm{U}^{\mathrm{T}}$. The left adjoint $\mathrm{F}^{\mathbf{T}}$ to $\mathrm{U}^{\mathrm{T}}$ is given by $\mathrm{F}^{\mathrm{T}} \mathrm{X}=\left(\mathrm{T}^{2} \mathrm{X} \stackrel{\mu_{\mathrm{X}}}{\longrightarrow} \mathrm{TX}\right)$ and the right adjoint $\mathrm{C}^{\mathrm{G}}$ to $\mathrm{V}^{\mathrm{G}}$ is given by $\mathrm{C}^{\mathrm{G}} \mathrm{X}=\left(\mathrm{TX} \stackrel{\delta_{\mathrm{X}}}{\longrightarrow} \mathrm{T}^{2} \mathrm{X}\right)$. Since $\mathrm{T} \mu \cdot \rho \mathrm{T}=\delta$, we see that $\mu$ and $\delta$ are mates as required to prove that $\mathrm{KF}^{\mathbf{T}}=\mathrm{C}^{\mathbf{G}}$. Since $\mathrm{V}^{\mathbf{G}} \dashv \mathrm{C}^{\mathbf{G}}$ with counit $\varepsilon$, we have $\mathrm{V}^{\mathrm{G}} \mathrm{K} \dashv \mathrm{K}^{-1} \mathrm{C}^{\mathrm{G}}$ with counit $\varepsilon$; that is, $\mathrm{U}^{\mathrm{T}} \dashv \mathrm{F}^{\mathrm{T}}$ with counit $\varepsilon$. QED

Proposition 1.5 Suppose $\mathrm{F} \dashv \mathrm{U} \dashv \mathrm{F}$ (written $\mathrm{F} \longmapsto \mathrm{U}$ ). Then the monad $\mathbf{T}$ generated by the adjunction $\mathrm{F} \rightarrow \mathrm{U}$ together with the counit for $\mathrm{U} \dashv \mathrm{F}$ is Frobenius.

Proof Let $\lambda:$ FU $\longrightarrow 1$ be the counit and $\eta: 1 \longrightarrow U F$ be the unit for $F \multimap$ U. Let $\varepsilon: \mathrm{UF} \longrightarrow 1$ be the counit and $\kappa: 1 \longrightarrow$ FU be the unit for $U \dashv$ F. The multiplication for $\mathbf{T}$ is $\mu=\mathrm{U} \lambda \mathrm{F}$. Take $\rho=\mathrm{U \kappa F} \circ \eta: 1 \longrightarrow \mathrm{T}^{2}$. Then $\mathrm{T}$ with $\varepsilon$ is Frobenius since

$$
\begin{gathered}
\mathrm{T} \mu \circ \rho \mathrm{T}=\mathrm{UFU} \lambda \mathrm{F} \circ \mathrm{U} \kappa \mathrm{FUF} \circ \eta \mathrm{UF}=\mathrm{U} \kappa \mathrm{F} \circ \mathrm{U} \lambda \mathrm{F} \circ \eta \mathrm{UF}=\mathrm{U} \kappa \mathrm{F} \\
\quad=\mathrm{U} \kappa \mathrm{F} \circ \mathrm{U} \lambda \mathrm{F} \circ \mathrm{UF} \eta=\mathrm{U} \lambda \mathrm{FUF} \circ \mathrm{UFU} \kappa \mathrm{F} \circ \mathrm{UF} \eta=\mu \mathrm{T} \circ \mathrm{T} \rho,
\end{gathered}
$$




$$
\begin{aligned}
& T \varepsilon \circ \rho=U F \varepsilon \circ U \kappa F \circ \eta=\eta, \quad \text { and } \\
& \varepsilon T \circ \rho=\varepsilon U F \circ U \kappa F \circ \eta=\eta . \quad \text { QED }
\end{aligned}
$$

See [Frd] for a discussion of $\mathrm{F} \longmapsto U$ in the special case where inter alia $U$ is fully faithful.

Theorem 1.6 Suppose $\mathbf{T}=(\mathrm{T}, \eta, \mu)$ is a monad on a category $X$ and suppose $\varepsilon: \mathrm{T} \longrightarrow 1$ is $a$ natural transformation. Then the following conditions are equivalent:

(a) equipped with $\varepsilon$, the monad $\mathbf{T}$ is Frobenius;

(b) there exists a natural transformation $\delta: \mathrm{T} \longrightarrow \mathrm{TT}$ such that

$$
\mathrm{T} \mu \circ \delta \mathrm{T}=\delta \circ \mu=\mu \mathrm{T} \circ \mathrm{T} \delta \quad \text { and } \mathrm{T} \varepsilon \circ \delta=1_{\mathrm{T}}=\varepsilon \mathrm{T} \circ \delta ;
$$

(c) there exists a comonad $\mathrm{G}=(\mathrm{T}, \varepsilon, \delta)$ such that

$$
\mathrm{T} \mu \circ \delta \mathrm{T}=\delta \circ \mu=\mu \mathrm{T} \circ \mathrm{T} \delta ;
$$

(d) there exists a counit $\sigma: \mathrm{T}^{2} \longrightarrow 1$ for an adjunction $\mathrm{T} \longrightarrow \mathrm{T}$ satisfying the equation

$$
\sigma \circ \mathrm{T} \mu=\sigma \circ \mu \mathrm{T}
$$

where $\varepsilon=\sigma \circ \eta \mathrm{T}$;

(e) the natural transformation $\sigma=\varepsilon \circ \mu: \mathrm{T}^{2} \longrightarrow 1$ is a counit for an adjunction $\mathrm{T} \mapsto \mathrm{T}$;

(f) the functor $\mathrm{F}^{\mathbf{T}}: X \longrightarrow X^{\mathbf{T}}$ is right adjoint to $\mathrm{U}^{\mathbf{T}}: X^{\mathbf{T}} \longrightarrow X$ with counit $\varepsilon$.

\section{Proof Equivalence of (a), (b) and (c).}

We have proved that (a) implies (b) and (c). Clearly (c) implies (b). To see that (b) implies (a), put $\rho=\delta \circ \eta$. Then

$$
\begin{gathered}
\mathrm{T} \mu \circ \rho \mathrm{T}=\mathrm{T} \mu \circ \delta \mathrm{T} \circ \eta \mathrm{T}=\delta \circ \mu \circ \eta \mathrm{T}=\delta=\delta \circ \mu \circ \mathrm{T} \eta=\mu \mathrm{T} \circ \mathrm{T} \delta \circ \mathrm{T} \eta=\mu \mathrm{T} \circ \mathrm{T} \rho \text { and } \\
\mathrm{T} \varepsilon \circ \rho=\mathrm{T} \varepsilon \circ \delta \circ \eta=\eta=\varepsilon \mathrm{T} \circ \delta \circ \eta=\varepsilon \mathrm{T} \circ \rho .
\end{gathered}
$$

Equivalence of (a) and (d).

Assuming (a), we know that $\sigma=\varepsilon \circ \mu: \mathrm{T}^{2} \longrightarrow 1$ is a counit for $\mathrm{T} \dashv \mathrm{T}$ by (b). But then $\sigma \circ \mathrm{T} \mu=\sigma \circ \mu \mathrm{T}$ is obvious by associativity of $\mu$.

Assume $(\mathrm{d})$ and note that $\sigma \circ \mathrm{T} \mu=\sigma \circ \mu \mathrm{T}$, in string notation, becomes:
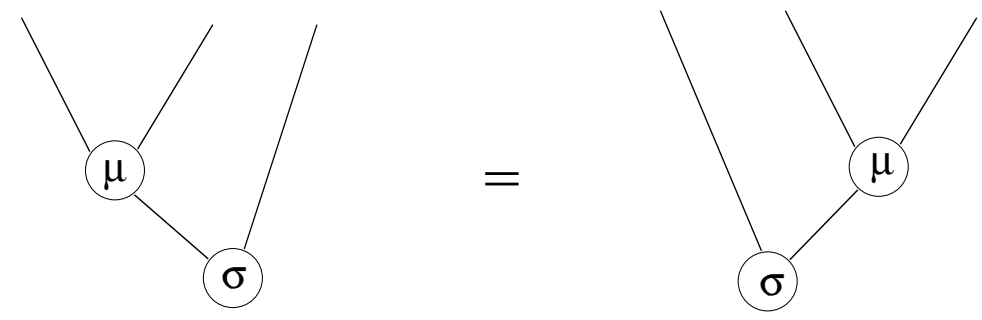

Let $\rho$ be the unit corresponding to the counit $\sigma$. The following proves $\mathrm{T} \mu \circ \rho \mathrm{T}=\mu \mathrm{T} \circ \mathrm{T} \rho$. 


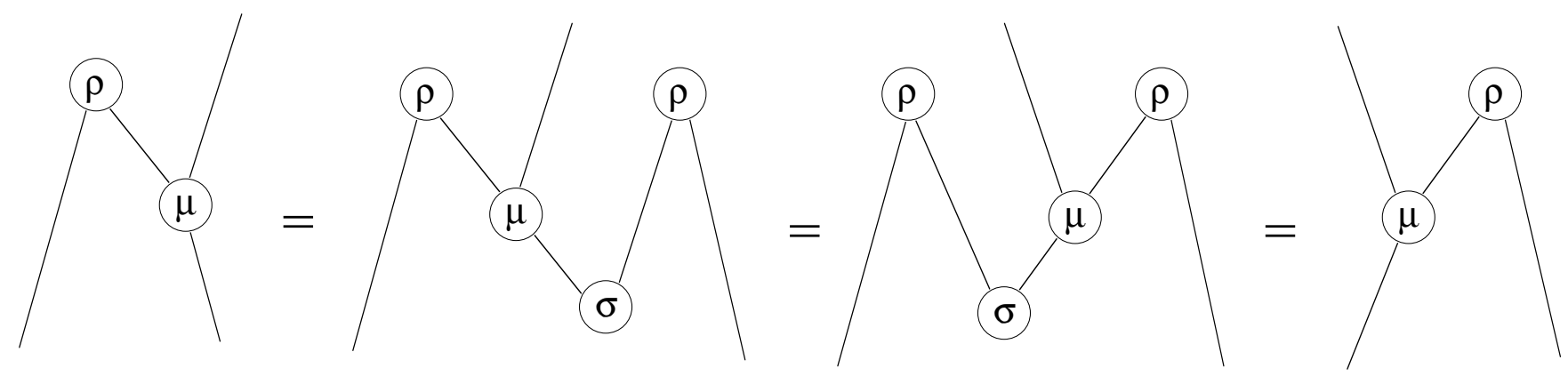

Now notice that $\sigma \circ \eta \mathrm{T}=\sigma \circ \mathrm{T} \eta$ by the following calculation.

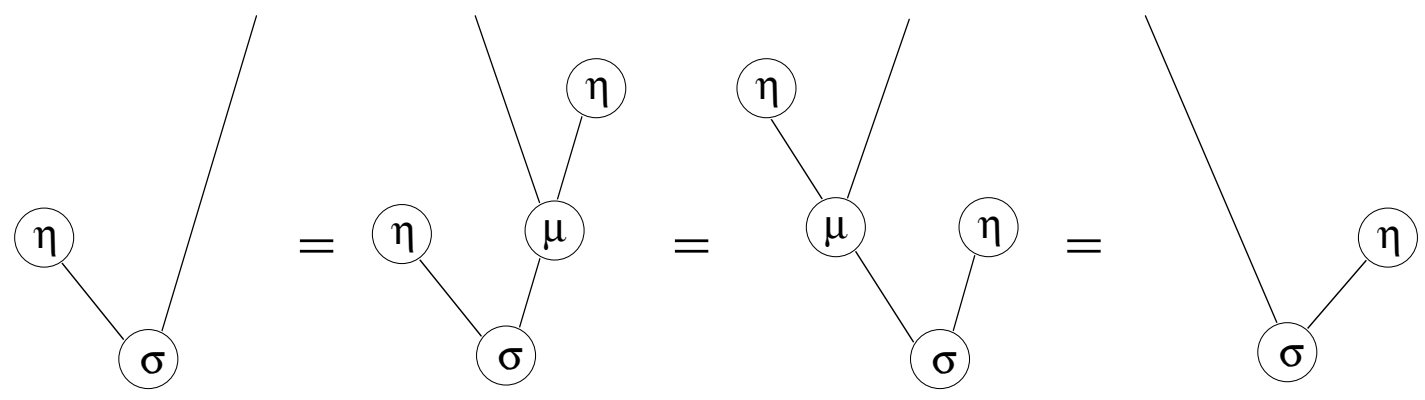

So put $\varepsilon=\sigma \circ \eta \mathrm{T}=\sigma \circ \mathrm{T} \eta$ and notice that

$$
\mathrm{T} \varepsilon \circ \rho=\mathrm{T} \sigma \circ \mathrm{T}^{2} \eta \circ \rho=\mathrm{T} \sigma \circ \rho \mathrm{T} \circ \eta=\eta=\sigma \mathrm{T} \circ \mathrm{T} \rho \circ \eta=\sigma \mathrm{T} \circ \eta \mathrm{T}^{2} \circ \rho=\varepsilon \mathrm{T} \circ \rho .
$$

So (a) holds.

Equivalence of (a) and (e).

Lemma 1.3 provides one direction. Conversely, if (e) holds then (d) holds since $\sigma \circ \mathrm{T} \mu=\sigma \circ \mu \mathrm{T}$ by associativity of $\mu$. So (a) holds.

Equivalence of (a) and (f).

This is an immediate consequence of Propositions 1.4 and 1.5. QED

It is clear from Theorem 1.6 that our definition agrees with Lawvere's definition of Frobenius monad (see pages 151 and 152 of [Law1]). Using the "algebra" terminology, it also agrees for example with Chapter 5 of [Cmd], Section 6 of [BS], and Definition 3.1 of [Mü].

It follows also that the notion of Frobenius monad is self-dual in the sense that it is the same as a comonad $G=(G, \varepsilon, \delta)$ with a natural transformation $\eta: 1_{\chi} \longrightarrow G$ such that $\delta \circ \eta$ is a unit for $G \dashv G$.

\section{§2. Review of enriched categories}

References for enriched categories are [Kel] and [Law2]. Let $\mathcal{V}$ denote a particularly familiar symmetric monoidal category. The reader really only needs to keep in mind the category Set of sets with cartesian product as tensor product and the category Vect of $_{\mathrm{k} \text { - }}$ linear spaces with usual tensor product. There are other nice examples such as Cat with cartesian product or the category DGVect $_{\mathrm{k}}$ of chain complexes of (that is, differential graded) vector spaces.

We call $\mathcal{V}$ our base monoidal category. A category $\mathcal{A}$ enriched in the base $\mathcal{V}$, also called a 
$\mathcal{V}$-category, consists of:

(i) a set $\operatorname{ob} \mathcal{A}$ whose elements are called objects;

(ii) for each pair of objects $\mathrm{A}$ and $\mathrm{B}$ of $\mathcal{A}$, an object $\mathcal{A}(\mathrm{A}, \mathrm{B})$ of $\mathcal{V}$ (that some people write as $\left.\operatorname{Hom}_{\mathcal{A}}(\mathrm{A}, \mathrm{B})\right)$;

(iii) morphisms $\mathcal{A}(\mathrm{B}, \mathrm{C}) \otimes \mathcal{A}(\mathrm{A}, \mathrm{B}) \longrightarrow \mathcal{A}(\mathrm{A}, \mathrm{C})$ in $\mathcal{V}$ called composition; and

(iv) morphisms $\mathrm{I} \longrightarrow \mathcal{A}(\mathrm{A}, \mathrm{A})$ in $\mathcal{V}$ called identity morphisms of $\mathcal{A}$;

subject to associativity and unital conditions. There is an underlying ordinary category whose objects are the same as those of $\mathcal{A}$ and whose morphisms $\mathrm{f}: \mathrm{A} \longrightarrow \mathrm{B}$ (we call them morphisms of $\mathcal{A}$ ) are morphisms $\mathrm{f}: \mathrm{I} \longrightarrow \mathcal{A}(\mathrm{A}, \mathrm{B})$ in $\mathcal{V}$. Each object $\mathrm{A}$ of $\mathcal{A}$ determines a monoid $\mathcal{A}(\mathrm{A}, \mathrm{A})$ in $\mathcal{V}$. Each monoid $\mathrm{M}$ in $\mathcal{V}$ determines a $\mathcal{V}$-category $\Sigma \mathrm{M}$ with one object whose endohom is M. Taking the other view, we can think of $\mathcal{V}$-categories as "monoids in $\mathcal{V}$ with several objects".

When $\mathcal{V}=$ Set, a $\mathcal{V}$-category is a category. When $\mathcal{V}=$ Vect $_{\mathrm{k}}$, a $\mathcal{V}$-category is a k-linear category (this is simply an ordinary category whose homs are k-vector spaces and whose composition is bilinear). Each k-algebra $\mathrm{E}$ determines a Vect $_{\mathrm{k}}$-category $\Sigma \mathrm{E}$ with one object. A 2-category is a Cat-category!

The nice bases $\mathcal{V}$ we have in mind are closed: for each pair of objects $\mathrm{X}$ and $\mathrm{Y}$ of $\mathcal{V}$ there is an object $[\mathrm{X}, \mathrm{Y}]$ (called the internal hom of $\mathrm{X}$ and $\mathrm{Y}$ ) and a natural bijection

$$
\mathcal{V}(\mathrm{W} \otimes \mathrm{X}, \mathrm{Y}) \cong \mathcal{V}(\mathrm{W},[\mathrm{X}, \mathrm{Y}]) \text {. }
$$

In this case $\mathcal{V}$ itself becomes a $\mathcal{V}$-category; so sometimes we write $\mathcal{V}(\mathrm{X}, \mathrm{Y})$ for the object $[X, Y]$ rather than merely its underlying set. In a non-symmetric monoidal category $\mathcal{V}$ this $[\mathrm{X}, \mathrm{Y}]$ would be called a left internal hom. A right internal hom $[\mathrm{X}, \mathrm{Y}]_{\mathrm{r}}$ satisfies

$$
\mathcal{V}(\mathrm{X} \otimes \mathrm{W}, \mathrm{Y}) \cong \mathcal{V}\left(\mathrm{W},[\mathrm{X}, \mathrm{Y}]_{\mathrm{r}}\right) .
$$

In any monoidal category $\mathcal{V}$ then, if $\mathrm{U}$ is right dual to $\mathrm{V}$, then there is a right internal hom of $\mathrm{V}$ and any $\mathrm{Y}$ given by $[\mathrm{V}, \mathrm{Y}]_{\mathrm{r}}=\mathrm{U} \otimes \mathrm{Y}$.

The opposite $\mathcal{A}^{\text {op }}$ of a $\mathcal{V}$-category $\mathcal{A}$ is the $\mathcal{V}$-category with the same objects as $\mathcal{A}$ and with $\mathcal{A}^{\mathrm{op}}(\mathrm{A}, \mathrm{B})=\mathcal{A}(\mathrm{B}, \mathrm{A})$; the composition uses that of $\mathcal{A}$ but "in the reverse order" as allowed by the symmetry of $\mathcal{V}$. We will see that, in the appropriate context, $\mathcal{A}^{\text {op }}$ is a dual of $\mathcal{A}$ in the same way that for finite-dimensional vector spaces $\mathrm{V}$, the space $\mathrm{V}^{*}$ of linear functionals is a dual of $\mathrm{V}$.

The definition of $\mathcal{V}$-functor $\mathrm{T}: \mathcal{A} \longrightarrow \mathcal{B}$ between $\mathcal{V}$-categories $\mathcal{A}$ and $\mathcal{B}$ is defined in the obvious way: it consists of a function $\mathrm{T}: \mathrm{ob} \mathcal{A} \longrightarrow \mathrm{ob} \mathcal{B}$ and morphisms 


$$
\mathrm{T}=\mathrm{T}_{\mathrm{A}, \mathrm{B}}: \mathcal{A}(\mathrm{A}, \mathrm{B}) \longrightarrow \mathcal{B}(\mathrm{TA}, \mathrm{TB})
$$

in $\mathcal{V}$ (called the effect of $\mathrm{T}$ on homs) compatible with composition and identities. The definition of $\mathcal{V}$-natural transformation $\theta: \mathrm{T} \Rightarrow \mathrm{T}^{\prime}: \mathcal{A} \longrightarrow \mathcal{B}$ needs a little more care in general, however, for the cases $\mathcal{V}=$ Set and $\mathcal{V}=$ Vect $_{\mathrm{k}}$, it amounts to an ordinary natural transformation.

With compositions that are straightforward (especially in the examples), we obtain a 2category $\mathcal{V}$-Cat whose objects are (small) $\mathcal{V}$-categories, whose morphisms are $\mathcal{V}$-functors and whose 2-cells are $\mathcal{V}$-natural transformations. As a technical point concerning size, we allow ourselves to write $\mathcal{V}$-Cat $(\mathcal{A}, \mathcal{B})$ for the category of $\mathcal{V}$-functors from $\mathcal{A}$ to $\mathcal{B}$ even when $\mathcal{A}$ and $\mathcal{B}$ are not small.

There is a tensor product $\mathcal{A} \otimes \mathcal{B}$ of $\mathcal{V}$-categories with $\mathrm{ob}(\mathcal{A} \otimes \mathcal{B})=\mathrm{ob} \mathcal{A} \times \mathrm{ob} \mathcal{B}$ and

$$
(\mathcal{A} \otimes \mathcal{B})\left((\mathrm{A}, \mathrm{B}),\left(\mathrm{A}^{\prime}, \mathrm{B}^{\prime}\right)\right)=\mathcal{A}\left(\mathrm{A}, \mathrm{A}^{\prime}\right) \otimes \mathcal{B}\left(\mathrm{B}, \mathrm{B}^{\prime}\right) ;
$$

the composition uses the compositions of $\mathcal{A}$ and $\mathcal{B}$ and the symmetry of $\mathcal{V}$. The unit for this tensor product is the $\mathcal{V}$-category $I$ with one object, denoted by $\bullet$, and with $I(\bullet, \bullet)=I$. Equipped with this, $\mathcal{V}$-Cat becomes a symmetric monoidal 2-category. Actually it is also closed; we now describe the internal hom.

For $\mathcal{V}$-categories $\mathcal{A}$ and $\mathcal{B}$, there is a $\mathcal{V}$-functor category $[\mathcal{A}, \mathcal{B}]$ : it is the $\mathcal{V}$-category whose objects are $\mathcal{V}$-functors from $\mathcal{A}$ to $\mathcal{B}$ and whose homs are given by the ends

$$
[\mathcal{A}, \mathcal{B}]\left(\mathrm{T}, \mathrm{T}^{\prime}\right)=\int_{\mathrm{A}} \mathcal{B}\left(\mathrm{TA}, \mathrm{T}^{\prime} \mathrm{A}\right)
$$

(see [McL] for Yoneda-Day-Kelly integral notation). In the examples of bases of interest here, it is clear that the $\mathcal{V}$-natural transformations from $\mathrm{T}$ to $\mathrm{T}^{\prime}$ form an object of $\mathcal{V}$. There is a canonical isomorphism of categories

$$
\mathcal{V} \text { - Cat }(\mathcal{C},[\mathcal{A}, \mathcal{B}]) \cong \mathcal{V} \text { - Cat }(C \otimes \mathcal{A}, \mathcal{B})
$$

There is another monoidal bicategory $\mathcal{V}$-Mod whose objects are also the (small) $\mathcal{V}$ categories; in this case however, the hom-categories are defined by

$$
\mathcal{V} \text { - } \operatorname{Mod}(\mathcal{A}, \mathcal{B})=\mathcal{V} \text {-Cat }\left(\mathcal{B}^{\mathrm{op}} \otimes \mathcal{A}, \mathcal{V}\right) \text {. }
$$

In other words, the morphisms of $\mathcal{V}$-Mod are $\mathcal{V}$-functors $\mathrm{M}: \mathcal{B}^{\mathrm{op}} \otimes \mathcal{A} \longrightarrow \mathcal{V}$ and the 2-cells are $\mathcal{V}$-natural transformations. These objects $\mathrm{M}$ of $\mathcal{V}$-Mod $(\mathcal{A}, \mathcal{B})$ are called modules from $\mathcal{A}$ to $\mathcal{B}$ (or "left $\mathcal{A}$-, right $\mathcal{B}$-bimodules") because of the actions ${ }^{1}$

$$
\mathcal{A}\left(\mathrm{A}, \mathrm{A}^{\prime}\right) \otimes \mathrm{M}(\mathrm{B}, \mathrm{A}) \otimes \mathcal{B}\left(\mathrm{B}^{\prime}, \mathrm{B}\right) \longrightarrow \mathrm{M}\left(\mathrm{B}^{\prime}, \mathrm{A}^{\prime}\right)
$$

that correspond to the effect of $\mathrm{M}$ on homs. The composite of modules $\mathrm{M}: \mathcal{A} \longrightarrow \mathcal{B}$ and $\mathrm{N}: \mathcal{B} \longrightarrow \mathcal{C}$ is defined by the coend 


$$
(\mathrm{N} \circ \mathrm{M})(\mathrm{C}, \mathrm{A})=\int^{\mathrm{B}} \mathrm{M}(\mathrm{B}, \mathrm{A}) \otimes \mathrm{N}(\mathrm{C}, \mathrm{B})
$$

this colimit can be constructed by taking a sum (coproduct) in $\mathcal{V}$ over all objects B and factoring out the left action of $\mathcal{B}$ on $\mathrm{N}$ and the right action of $\mathcal{B}$ on $\mathrm{M}$ (instead of a composition we can think of it as a tensor product over $\mathcal{B}$ : in that spirit we can write $\mathrm{N} \circ \mathrm{M}=\mathrm{M} \underset{\mathcal{B}}{\otimes} \mathrm{N}$ ). This composition (like tensor product) is only associative up to isomorphism (which is allowed in a bicategory). The identity module of $\mathcal{A}$ is the module $1_{\mathcal{A}}$ defined by $1_{\mathcal{A}}\left(\mathrm{A}, \mathrm{A}^{\prime}\right)=\mathcal{A}\left(\mathrm{A}, \mathrm{A}^{\prime}\right)$.

Each $\mathcal{V}$-functor $\mathrm{T}: \mathcal{A} \longrightarrow \mathcal{B}$ yields a modules $\mathrm{T}_{*}: \mathcal{A} \longrightarrow \mathcal{B}$ and $\mathrm{T}^{*}: \mathcal{B} \longrightarrow \mathcal{A}$ defined by $\mathrm{T}_{*}(\mathrm{~B}, \mathrm{~A})=\mathcal{B}(\mathrm{B}, \mathrm{TA}) \quad$ and $\quad \mathrm{T}^{*}(\mathrm{~A}, \mathrm{~B})=\mathcal{B}(\mathrm{TA}, \mathrm{B})$. In fact, $\mathrm{T}^{*}$ is right adjoint to $\mathrm{T}_{*}$ in the bicategory $\mathcal{V}$-Mod: the unit $1_{\mathcal{A}} \longrightarrow \mathrm{T}^{*} \circ \mathrm{T}_{*}$ has components

$$
\mathcal{A}\left(\mathrm{A}, \mathrm{A}^{\prime}\right) \longrightarrow \int^{\mathrm{B}} \mathcal{B}(\mathrm{TA}, \mathrm{B}) \otimes \mathcal{B}\left(\mathrm{B}, \mathrm{TA}^{\prime}\right)=\mathcal{B}\left(\mathrm{TA}, \mathrm{TA}^{\prime}\right)
$$

given by the effect of $\mathrm{T}$ on homs. We obtain an "inclusion" pseudofunctor

$$
\mathcal{V} \text { - Cat } \longrightarrow \mathcal{V} \text {-Mod }
$$

that takes each $\mathcal{V}$-category to itself, takes each $\mathcal{V}$-functor $\mathrm{T}$ to $\mathrm{T}_{*}$, and is bijective on 2-cells; so we sometimes write $\mathrm{T}$ for the module $\mathrm{T}_{*}$.

So we can think of $\mathcal{V}$-Mod as an expansion of $\mathcal{V}$-Cat designed to provide the morphisms in $\mathcal{V}$-Cat with right adjoints. Not only adjoints but equivalences in $\mathcal{V}$-Mod are also of interest.

Equivalence in $\mathcal{V}$-Mod is called Cauchy equivalence of $\mathcal{V}$-categories. In the particular case where $\mathcal{V}$ is the monoidal category of abelian groups with the usual tensor product, we obtain the notion of Cauchy equivalence for additive categories. Rings $\mathrm{R}$ can be regarded as additive categories $\Sigma \mathrm{R}$ with a single object; in this way, Cauchy equivalence is none other than ordinary Morita equivalence of rings.

Each $\mathcal{V}$-category $\mathcal{A}$ has a Cauchy completion $Q \mathcal{A}:$ it is the smallest full sub- $\mathcal{V}$-category of the $\mathcal{V}$-functor $\mathcal{V}$-category $\mathscr{P} \mathcal{A}=\left[\mathcal{A}^{\text {op }}, \mathcal{V}\right]$ that contains the representable $\mathcal{V}$-functors $\mathcal{A}(-, \mathrm{A})$ and is closed under absolute $\mathcal{V}$-colimits. (Absolute $\mathcal{V}$-colimits are those preserved by all $\mathcal{V}$ functors; see [St3].) For example, if $\mathcal{V}=$ Set then $Q \mathcal{A}$ is the completion of the category $\mathcal{A}$ under splitting of idempotents; and if $\mathcal{V}=\operatorname{Vect}_{\mathrm{k}}$ then $Q \mathcal{A}$ is the completion of the additive category $\mathcal{A}$ under direct sums and splitting of idempotents.

It is easy to see that $\mathcal{V}$-categories $\mathcal{A}$ and $\mathcal{B}$ are Cauchy equivalent if and only if $\mathcal{P} \mathcal{A}$ and $\mathcal{P B}$ are equivalent $\mathcal{V}$-categories (that is, equivalent in the 2 -category $\mathcal{V}$-Cat). It is well known (see [St2] for a proof in a very general context) that $\mathcal{V}$-categories $\mathcal{A}$ and $\mathcal{B}$ are Cauchy 
equivalent if and only if $Q \mathcal{A}$ and $Q \mathcal{B}$ are equivalent $\mathcal{V}$-categories. The inclusion of $\mathrm{Q} \mathcal{A}$ in $Q Q \mathcal{A}$ is an equivalence, so $\mathcal{A}$ is Cauchy equivalent to $\mathcal{Q} \mathcal{A}$. We say $\mathcal{A}$ is Cauchy complete when it admits all absolute $\mathcal{V}$-colimits; that is, when the inclusion of $\mathcal{A}$ in $Q \mathcal{A}$ is an equivalence.

Tensor product of $\mathcal{V}$-categories extends to $\mathcal{V}$-Mod making $\mathcal{V}$-Mod a symmetric monoidal bicategory and the inclusion of $\mathcal{V}$-Cat in $\mathcal{V}$-Mod strict monoidal. We have seen that $\mathcal{V}$-Cat is closed. However, there is a much stronger structure possessed by $\mathcal{V}$-Mod; it is "autonomous" like the category of finite-dimensional vector spaces, and we shall now make this precise.

We work in a monoidal bicategory $\mathcal{B}$. A morphism $\mathrm{e}: \mathrm{A} \otimes \mathrm{B} \longrightarrow \mathrm{I}$ is called a biexact pairing when the functor

$$
\mathcal{B}(\mathrm{C}, \mathrm{B} \otimes \mathrm{D}) \longrightarrow \mathcal{B}(\mathrm{A} \otimes \mathrm{C}, \mathrm{D})
$$

taking $\mathrm{C} \stackrel{\mathrm{f}}{\longrightarrow} \mathrm{B} \otimes \mathrm{D}$ to $\mathrm{A} \otimes \mathrm{C} \stackrel{\mathrm{A} \otimes \mathrm{f}}{\longrightarrow} \mathrm{A} \otimes \mathrm{B} \otimes \mathrm{D} \stackrel{\mathrm{e} \otimes \mathrm{D}}{\longrightarrow} \mathrm{D}$, is an equivalence of categories for all objects $C$ and $D$. In this case, there is a unique (up to isomorphism) morphism $\mathrm{n}: \mathrm{I} \longrightarrow \mathrm{B} \otimes \mathrm{A}$ such that the composite $\mathrm{A} \stackrel{\mathrm{A} \otimes \mathrm{n}}{\longrightarrow} \mathrm{A} \otimes \mathrm{B} \otimes \mathrm{A} \stackrel{\mathrm{e} \otimes \mathrm{A}}{\longrightarrow} \mathrm{A}$ is isomorphic to the identity of $\mathrm{A}$. We say that $\mathrm{B}$ is a right bidual for $\mathrm{A}$ with counit $\mathrm{e}$ and unit $\mathrm{n}$. Of course, A is called a left bidual for B.

The monoidal bicategory $\mathcal{B}$ is called autonomous when each object has both a left and a right bidual. A choice of right bidual of an object $A$ is denoted by $A^{\circ}$ : with unit $\mathrm{n}: \mathrm{I} \longrightarrow \mathrm{A}^{\circ} \otimes \mathrm{A}$ and counit $\mathrm{e}: \mathrm{A} \otimes \mathrm{A}^{\circ} \longrightarrow \mathrm{I}$.

In the case of $\mathcal{V}$-Mod, a right bidual for the $\mathcal{V}$-category $\mathcal{A}$ is provided by $\mathcal{A}^{\text {op }}$ since

$$
\mathcal{V}-\operatorname{Mod}(\mathcal{A} \otimes \mathcal{B}, \mathcal{C}) \cong \mathcal{V} \text { - Cat }\left(\mathcal{C}^{\mathrm{op}} \otimes \mathcal{A} \otimes \mathcal{B}, \mathcal{V}\right) \cong \mathcal{V}-\operatorname{Mod}\left(\mathcal{B}, \mathcal{A}^{\mathrm{op}} \otimes \mathcal{C}\right) .
$$

The counit $\mathrm{e}: \mathcal{A} \otimes \mathcal{A}^{\mathrm{op}} \longrightarrow I$ is the module defined by $\mathrm{e}\left(\bullet, \mathrm{A}, \mathrm{A}^{\prime}\right)=\mathcal{A}\left(\mathrm{A}^{\prime}, \mathrm{A}\right)$. Since $\mathcal{V}$-Mod is symmetric, it is autonomous.

\section{§3. Frobenius pseudomonoids}

We consider a pseudomonoid (or monoidal object) $\mathrm{A}$ in a monoidal bicategory $\mathcal{B}$ : the underlying object is also denoted by $A$, the unit is $\mathrm{j}: \mathrm{I} \longrightarrow \mathrm{A}$, the multiplication is $\mathrm{p}: \mathrm{A} \otimes \mathrm{A} \longrightarrow \mathrm{A}$, and there are invertible coherent associativity and unital constraints. When the unit constraints are identities, the pseudomonoid is said to be normalized. When the unit and associativity constraints are identities, the pseudomonoid is said to be strict; it is then just a monoid.

Motivated by Theorem 1.6 (e) we make a natural higher-dimensional extension of the Frobenius notion. 
Definition 3.1 A pseudomonoid A is Frobenius when it is equipped with a morphism $\ell: \mathrm{A} \longrightarrow \mathrm{I}$ such that the composite

$$
\mathrm{A} \otimes \mathrm{A} \stackrel{\mathrm{p}}{\longrightarrow} \mathrm{A} \stackrel{\ell}{\longrightarrow} \mathrm{I}
$$

is a biexact pairing.

Proposition 1.1 of [DMS] states that the pseudomonoid A is left autonomous with dualization $\mathrm{d}: \mathrm{A}^{\circ} \longrightarrow \mathrm{A}$ if and only if

$$
\mathrm{p}^{*} \cong(\mathrm{p} \otimes \mathrm{A}) \circ(\mathrm{A} \otimes \mathrm{d} \otimes \mathrm{A}) \circ(\mathrm{A} \otimes \mathrm{n}),
$$

and this holds if and only if

$$
(\mathrm{p} \circ(\mathrm{d} \otimes A))^{*} \cong\left(\mathrm{A}^{\circ} \otimes \mathrm{p}\right) \circ(\mathrm{n} \otimes A) .
$$

Proposition 1.2 of [DMS] gives the formula

$$
\mathrm{d} \cong(\mathrm{A} \otimes \mathrm{e}) \circ\left(\mathrm{p}^{*} \otimes \mathrm{A}^{\circ}\right) \circ\left(\mathrm{j} \otimes \mathrm{A}^{\circ}\right) .
$$

Furthermore, in the case where $\mathrm{j}$ has a right adjoint, Proposition 1.4 of [DMS] states that $\mathrm{A}$ is also right autonomous, and then simply called autonomous, if and only if $d$ is an equivalence. Then the right adjoint $\mathrm{d}^{*}$ of $\mathrm{d}$ is an inverse equivalence and (Proposition 1.2 of [DMS]) is given by the formula

$$
d^{*} \cong\left(A^{\circ} \otimes j^{*}\right) \circ\left(A^{\circ} \otimes p\right) \circ(n \otimes A) .
$$

Proposition 3.1 Every autonomous pseudomonoid $(\mathrm{A}, \mathrm{j}, \mathrm{p})$, for which $\mathrm{j}$ has a right adjoint, is Frobenius with $\ell=j^{*}$.

Proof In an autonomous monoidal bicategory, the composite $\sigma: \mathrm{A} \otimes \mathrm{A} \stackrel{\mathrm{p}}{\longrightarrow} \mathrm{A} \stackrel{\ell}{\longrightarrow} \mathrm{I}$ is a biexact pairing if and only if the corresponding morphism

$$
\hat{\sigma}: \mathrm{A} \stackrel{\mathrm{n} \otimes \mathrm{A}}{\longrightarrow} \mathrm{A}^{\circ} \otimes \mathrm{A} \otimes \mathrm{A} \stackrel{\mathrm{A}^{\circ} \otimes \sigma}{\longrightarrow} \mathrm{A}^{\circ}
$$

is an equivalence. So, with $\ell=\mathrm{j}^{*}$, we have

$$
\hat{\sigma}=\left(A^{\circ} \otimes j^{*}\right) \circ\left(A^{\circ} \otimes p\right) \circ(n \otimes A) \cong d^{*},
$$

an equivalence. QED

A pseudocomonoidal structure on A in Proposition 3.1 is provided by $j^{*}$ and $p^{*}$; compare Theorem 1.6 (c). We also note that there are isomorphisms

$$
\mathrm{p}^{*} \cong(\mathrm{d} \otimes A) \circ\left(\mathrm{A}^{\circ} \otimes \mathrm{p}\right) \circ(\mathrm{n} \otimes A) \cong(\mathrm{A} \otimes \mathrm{p}) \circ(\rho \otimes A) \cong(\mathrm{p} \otimes A) \circ(A \otimes \rho)
$$

where $\rho=\left(\mathrm{I} \stackrel{\mathrm{j}}{\longrightarrow} \mathrm{A} \stackrel{\mathrm{p}^{*}}{\longrightarrow} \mathrm{A} \otimes \mathrm{A}\right)$.

Example Quasi-Hopfalgebras 
A quasibialgebra (over a field $\mathrm{k}$ ) is a $\mathrm{k}$-algebra $\mathrm{H}$ equipped with algebra morphisms

$$
\Delta: \mathrm{H} \longrightarrow \mathrm{H} \otimes \mathrm{H} \text { and } \mathrm{E}: \mathrm{H} \longrightarrow \mathrm{k},
$$

and with an invertible element $\phi \in \mathrm{H} \otimes \mathrm{H} \otimes \mathrm{H}$, such that

$$
\left(\mathrm{E} \otimes 1_{\mathrm{H}}\right)(\Delta(\mathrm{a}))=\mathrm{a}=\left(1_{\mathrm{H}} \otimes \mathrm{E}\right)(\Delta(\mathrm{a})) \quad \text { and } \quad \phi\left(\Delta \otimes 1_{\mathrm{H}}\right)(\Delta(\mathrm{a}))=\left(1_{\mathrm{H}} \otimes \Delta\right)(\Delta(\mathrm{a})) \phi
$$

for all $\mathrm{a} \in \mathrm{H}$; furthermore, $\phi$ satisfies the pentagon condition

$$
(1 \otimes 1 \otimes \Delta)(\phi) \cdot(\Delta \otimes 1 \otimes 1)(\phi)=(1 \otimes \phi) \cdot(1 \otimes \Delta \otimes 1)(\phi) \cdot(\phi \otimes 1) .
$$

(A quasibialgebra reduces to an ordinary bialgebra when $\phi$ is the identity element $1 \otimes 1 \otimes 1$.) We can make $\mathrm{H} \otimes \mathrm{H}$ into a left $\mathrm{H} \otimes \mathrm{H}$-, right $\mathrm{H}$-bimodule by means of the actions

$$
(a \otimes b) \cdot(x \otimes y) \cdot c=\sum_{i} a x c_{i}^{1} \otimes b y c_{i}^{2}
$$

where $\Delta(\mathrm{c})=\sum_{\mathrm{i}} \mathrm{c}_{\mathrm{i}}^{1} \otimes \mathrm{c}_{\mathrm{i}}^{2}$; for the time being, let us call this bimodule M. Given an algebra antimorphism $\mathrm{S}: \mathrm{H} \longrightarrow \mathrm{H}$, there is another left $\mathrm{H} \otimes \mathrm{H}-$, right $\mathrm{H}$-bimodule structure defined on $\mathrm{H} \otimes \mathrm{H}$ by the actions

$$
(a \otimes b) \cdot(x \otimes y) \cdot c=\sum_{j} a x S\left(b_{j}^{1}\right) \otimes b_{j}^{2} y c
$$

where $\Delta(\mathrm{b})=\sum_{\mathrm{j}} \mathrm{b}_{\mathrm{j}}^{1} \otimes \mathrm{b}_{\mathrm{j}}^{2}$; for the time being, let us call this bimodule N. A quasi-Hopfalgebra is a quasibialgebra $\mathrm{H}$ together with an algebra anti-morphism $\mathrm{S}: \mathrm{H} \longrightarrow \mathrm{H}$ (called the antipode) and a bimodule isomorphism $\pi: \mathrm{M} \cong \mathrm{N}$. This is equivalent to the original definition of [Dd] that, instead of the isomorphism $\pi$, involved two elements $\alpha$ and $\beta$ of $H$ satisfying the equations $E(c) \alpha=\sum_{i} S\left(c_{i}^{1}\right) \alpha c_{i}^{2}$ and $E(c) \beta=\sum_{i} c_{i}^{1} \beta S\left(c_{i}^{2}\right)$, and two further conditions involving $\alpha, \beta$ and $\phi$ (see Section 2.4 of [Maj]). We shall say a bit more about the equivalence of the definitions soon.

Take $\mathcal{V}=$ Vect $_{\mathrm{k}}$ and recall that a k-algebra $\mathrm{A}$ is a one-object $\mathcal{V}$-category $\Sigma \mathrm{A}$. It is obvious that an algebra morphism $\mathrm{f}: \mathrm{A} \longrightarrow \mathrm{B}$ is the same as a $\mathcal{V}$-functor $\mathrm{f}: \Sigma \mathrm{A} \longrightarrow \Sigma \mathrm{B}$. So what is a $\mathcal{V}$-natural transformation $\sigma: \mathrm{f} \Rightarrow \mathrm{g}$ in terms of the algebra morphisms $\mathrm{f}$ and $\mathrm{g}$ from $A$ to $B$ ? It is nothing other than an element $\alpha$ of $B$ such that $\alpha f(a)=g(a) \alpha$ for all elements a of $\mathrm{A}$. Therefore we see that $\phi$ belongs in the square

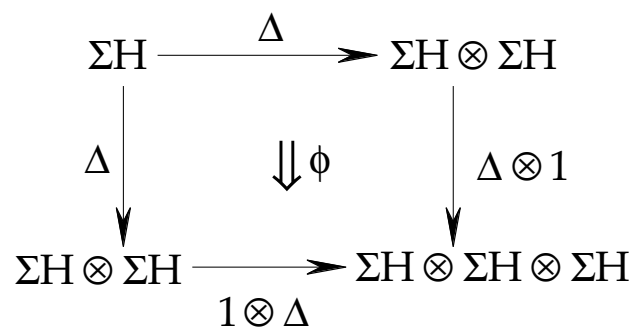


in $\mathcal{V}$-Cat. From this it follows easily that: a quasibialgebra structure on an algebra $\mathrm{H}$ is precisely a normalized pseudomonoid structure on $\Sigma \mathrm{H}$ in $(\mathcal{V} \text {-Cat })^{\text {coop }}$.

Now we move from $(\mathcal{V} \text {-Cat })^{\text {coop }}$ to $(\mathcal{V} \text {-Mod })^{\text {coop }}$ where $\mathcal{V}$-functors have right adjoints and the object $\Sigma \mathrm{H}^{\mathrm{op}}$ is a bidual for $\Sigma \mathrm{H}$. Here we can observe that the right adjoint $\Delta^{*}: \Sigma \mathrm{H} \otimes \Sigma \mathrm{H} \longrightarrow \Sigma \mathrm{H}$ of $\Delta$ is the bimodule $\mathrm{M}$ above and the composite module

$$
\Sigma \mathrm{H} \otimes \Sigma \mathrm{H} \stackrel{\Sigma \mathrm{H} \otimes \Delta}{\longrightarrow} \Sigma \mathrm{H} \otimes \Sigma \mathrm{H} \otimes \Sigma \mathrm{H} \stackrel{\Sigma \mathrm{H} \otimes \mathrm{S} \otimes \Sigma \mathrm{H}}{\longrightarrow} \Sigma \mathrm{H} \otimes \Sigma \mathrm{H}^{\mathrm{op}} \otimes \Sigma \mathrm{H} \stackrel{\mathrm{e} \otimes \Sigma \mathrm{H}}{\longrightarrow} \Sigma \mathrm{H}
$$

is $\mathrm{N}$. It follows that: a quasi-Hopf structure on the quasialgebra $\mathrm{H}$ is precisely a left autonomous structure on the normalized pseudomonoid $\Sigma \mathrm{H}$ in $(\mathcal{V} \text {-Mod })^{\text {coop }}$. Therefore Proposition 3.1 applies to yield a Frobenius structure on $\Sigma \mathrm{H}$ in $(\mathcal{V} \text {-Mod })^{\text {coop }}$ using $\mathrm{E}^{*}$. This means that $\Sigma \mathrm{H}$ becomes a pseudomonoid in $\mathcal{V}$-Mod using $\mathrm{E}^{*}$ and $\Delta^{*}$; of course, this is not the original algebra structure on $\mathrm{H}$.

Finally in this example, we can say something about the equivalent definitions of quasiHopf algebra: specifically about how $\pi$ is obtained from $\alpha$ and $\phi$. The above-mentioned equation satisfied by the element $\alpha$ of $\mathrm{H}$ says precisely that it is a (bi)module morphism from $\mathrm{E}$ to $\mathrm{e} \circ(1 \otimes S) \circ \Delta$ (note that $\mathrm{E}$ is $\mathrm{k}$ and $\mathrm{e} \circ(1 \otimes S) \circ \Delta$ is $\mathrm{H}$ with appropriate actions). Then $\pi$ is the pasting composite of the following diagram.

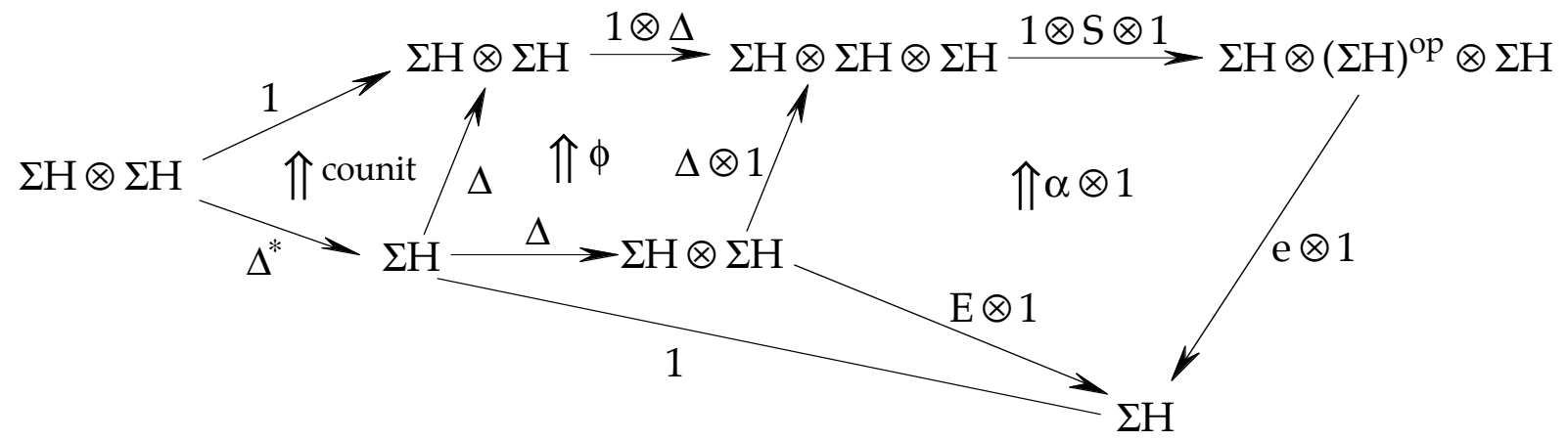

Similarly, $\beta$ is used to define a 2-cell in the opposite direction; the further conditions on $\alpha, \beta$ and $\phi$ say that this really is the inverse of $\pi$. Further details can be found in the general results of [DMS]. This ends our example.

In Section 9 of [DS2], a form for a pseudomonoid $\mathrm{A}$ in a monoidal bicategory $\mathcal{B}$ is defined to be a morphism $\sigma: \mathrm{A} \otimes \mathrm{A} \longrightarrow \mathrm{I}$ together with an invertible 2-cell $\gamma$ as below.

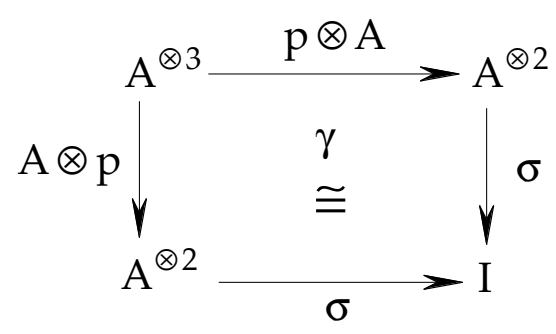


Then the authors define the pseudomonoid to be $*$-autonomous when $\sigma$ is a biexact pairing ${ }^{2}$.

Proposition 3.2 A pseudomonoid is *-autonomous if and only if it is Frobenius.

Proof Suppose $\sigma$ and $\gamma$ is a form on the pseudomonoid A with $\sigma$ a biexact pairing. Put $\ell=(\mathrm{A} \stackrel{\mathrm{j} \otimes \mathrm{A}}{\longrightarrow} \mathrm{A} \otimes \mathrm{A} \stackrel{\sigma}{\longrightarrow} \mathrm{I})$ so that

$$
\sigma \cong \sigma \circ(\mathrm{p} \otimes \mathrm{A}) \circ(\mathrm{j} \otimes \mathrm{A}) \cong \sigma \circ(\mathrm{A} \otimes \mathrm{p}) \circ(\mathrm{j} \otimes \mathrm{A}) \cong \sigma \circ(j \otimes A) \circ \mathrm{p} \cong \ell \circ \mathrm{p}
$$

where the second isomorphism involves $\gamma$. So A equipped with $\ell$ is Frobenius. Conversely, put $\sigma=\ell \circ \mathrm{p}$ which is a biexact pairing by definition of Frobenius; the isomorphism $\gamma$ is obtained by composing the associativity constraint

$$
\mathrm{p} \circ(\mathrm{p} \otimes \mathrm{A}) \cong \mathrm{p} \circ(\mathrm{A} \otimes \mathrm{p})
$$

on the left with $\ell$. QED

Corollary 3.3 For any object $\mathrm{X}$ of $\mathcal{B}$ and any equivalence $\mathrm{v}: \mathrm{X} \longrightarrow \mathrm{X}^{\circ \circ}$, the pseudomonoid $\mathrm{X}^{\circ} \otimes \mathrm{X}$ is Frobenius when equipped with $\ell: \mathrm{X}^{\circ} \otimes \mathrm{X} \stackrel{1 \otimes v}{\longrightarrow} \mathrm{X}^{\circ} \otimes \mathrm{X}^{\circ} \stackrel{\mathrm{e}}{\longrightarrow} \mathrm{I}$.

\section{Example Star-autonomous monoidal enriched categories}

A $\mathcal{V}$-category $\mathcal{A}$ is monoidal when it is equipped with the structure of pseudomonoid in $\mathcal{V}$-Cat; we write $\#: \mathcal{A} \otimes \mathcal{A} \longrightarrow \mathcal{A}$ for the "multiplication" $\mathcal{V}$-functor (to distinguish it from the tensor product $\otimes$ of $\mathcal{V}$ ) and $\mathrm{J}$ for the unit object. We say that $\mathcal{A}$ is *-autonomous when it is equipped with an equivalence of $\mathcal{V}$-categories $\mathrm{S}: \mathcal{A} \longrightarrow \mathcal{A}^{\text {op }}$ and a $\mathcal{V}$-natural family of isomorphisms

$$
\mathcal{A}(\mathrm{A} \# \mathrm{~B}, \mathrm{~J}) \cong \mathcal{A}(\mathrm{B}, \mathrm{SA}) .
$$

This is a straightforward enrichment of the concept due to Barr [Ba3] and considered more generally in [DS2] in connection with quantum groupoids. Here we want to emphasize the Frobenius aspect. In order to obtain an autonomous monoidal bicategory, we need to move from $\mathcal{V}$-Cat to $\mathcal{V}$-Mod. Because of the way we have defined $\mathcal{V}$-Mod, it is better to consider $\mathcal{A}^{\text {op }}$ rather than $\mathcal{A}$; to say one is monoidal is the same as saying the other is. For simplicity, we also suppose $\mathcal{A}$ is Cauchy complete (see Section 5); then any equivalence $\mathcal{A} \longrightarrow \mathcal{A}^{\text {op }}$ in $\mathcal{V}$-Mod is automatically of the form $\mathrm{S}_{*}$ for an equivalence $\mathrm{S}: \mathcal{A} \longrightarrow \mathcal{A}^{\text {op }}$ in $\mathcal{V}$ - Cat. As a consequence of Proposition 3.2 we have that: the monoidal $\mathcal{V}$-category $\mathcal{A}$ is $*$ autonomous if and only if $\mathcal{A}^{\mathrm{op}}$ is Frobenius in $\mathcal{V}$-Mod. If we write $\stackrel{\vee}{\mathrm{S}}: \mathcal{A}^{\mathrm{op}} \otimes \mathcal{A}^{\mathrm{op}} \longrightarrow I$ for the

\footnotetext{
${ }^{2}$ There was an extra condition in [DS2] requiring $\sigma$ to be "representable"; however, this is automatic under a mild completeness condition on the pseudomonoid.
} 
module defined by $\stackrel{\vee}{\mathrm{S}}(\mathrm{A}, \mathrm{B})=\mathcal{A}(\mathrm{B}, \mathrm{SA})$, we see that the isomorphism defining $*$-autonomy is precisely $\mathrm{J}^{*} \circ \#_{*} \cong \stackrel{\vee}{\mathrm{S}}$. Corollary 3.3 implies a result of [DS2] that, for any $\mathcal{V}$-category $x$, the monoidal $\mathcal{V}$-category $X^{\mathrm{op}} \otimes X$ is $*$-autonomous.

\section{$\S 4$. Projective equivalences}

This section was inspired to a large extent by the discussion of "weak monoidal Morita equivalence" in [Mü1] and [Mü2].

It is well known what it means for a morphism to be an equivalence in any bicategory and that every equivalence can be made an adjoint equivalence. We wish to discuss a more general notion of equivalence. For this we need to clarify a concept of scalar.

A scalar for a bicategory $\mathcal{D}$ is a modification

$$
\omega: 1_{1_{\mathcal{D}}} \longrightarrow 1_{1_{\mathcal{D}}}: 1_{\mathcal{D}} \longrightarrow 1_{\mathcal{D}}: \mathcal{D} \longrightarrow \mathcal{D} .
$$

That is, $\omega$ assigns to each object $\mathrm{A}$ of $\mathcal{D}$ a 2-cell $\omega_{\mathrm{A}}: 1_{\mathrm{A}} \longrightarrow 1_{\mathrm{A}}$ such that, for all morphisms $\mathrm{f}: \mathrm{A} \longrightarrow \mathrm{B}$,

$$
\mathrm{f} \omega_{\mathrm{A}}=\omega_{\mathrm{B}} \mathrm{f} \text {. }
$$

Scalars form a commutative monoid under composition. By abuse of language, 2-cell $\theta: \mathrm{f} \Rightarrow \mathrm{f}: \mathrm{X} \longrightarrow \mathrm{Y}$ will be called a scalar when there exists an actual scalar $\omega$ such that

$$
\theta=\mathrm{f} \omega_{X}
$$

we say $\theta$ is an invertible scalar when $\omega$ is invertible.

Take the example of enriched categories; that is, in the case where $\mathcal{D}=\mathcal{V}$-Mod. It is easy to see that the commutative monoid of scalars is isomorphic to the endomorphism monoid $\mathcal{V}(\mathrm{I}, \mathrm{I})$ of the unit object I. In particular, when $\mathcal{V}$ is the category of sets, the monoid is trivial (consisting only of the identity). More interestingly, if $\mathcal{V}$ is the monoidal category of modules over a commutative ring $\mathrm{k}$ then the scalars for $\mathcal{V}$-Mod are precisely the elements of $\mathrm{k}$.

Remark The braided monoidal category $\operatorname{Hom}(\mathcal{D}, \mathcal{D})\left(1_{\mathcal{D}}, 1_{\mathcal{D}}\right)$ whose objects are pseudonatural transformations of the identity of $\mathcal{D}$, whose morphisms are modifications, and whose tensor product is either of the two compositions, is called the centre of the bicategory $\mathcal{D}$. So scalars are endomorphisms of the unit of the centre. If $\mathcal{D}$ is the one-object bicategory $\Sigma \mathcal{C}$ with hom monoidal category $\mathcal{C}$ then $\operatorname{Hom}(\mathcal{D}, \mathcal{D})\left(1_{\mathcal{D}}, 1_{\mathcal{D}}\right)$ is the centre $Z \mathcal{C}$ of $\mathcal{C}$ in the sense of [JS2].

Definition 4.1 A morphism $\mathrm{u}: \mathrm{A} \longrightarrow \mathrm{X}$ in a bicategory $\mathcal{D}$ is called a projective equivalence when there is a morphism $\mathrm{f}: \mathrm{X} \longrightarrow \mathrm{A}$ adjoint to $\mathrm{u}$ on both sides (that is, $\mathrm{f} \longmapsto \mathrm{u}$ ) and 
there are invertible scalars $\omega$ and $\bar{\omega}$ such that the composites

$$
1_{\mathrm{X}} \stackrel{\eta}{\longrightarrow} \mathrm{u} \mathrm{f} \stackrel{\varepsilon}{\longrightarrow} 1_{\mathrm{X}} \text { and } 1_{\mathrm{A}} \stackrel{\kappa}{\longrightarrow} \mathrm{f} \mathrm{u} \stackrel{\lambda}{\longrightarrow} 1_{\mathrm{A}}
$$

are equal to $\omega_{X}$ and $\bar{A}_{A}$, respectively, where $\eta, \lambda$ and $\kappa, \varepsilon$ are the unit-counit pairs for the adjunctions.

In any projective equivalence, by suitable rescaling of units and counits, we can ensure that either $\omega$ or $\bar{\omega}$ is an identity. Equivalences are precisely the projective equivalences in which both $\omega$ and $\Phi$ can be chosen to be identities.

Proposition 4.1 A composite of projective equivalences is a projective equivalence.

Proof Suppose $\mathrm{f} \longmapsto \mathrm{u}$ with $\mathrm{u}: \mathrm{A} \longrightarrow \mathrm{X}$ and $\mathrm{f}^{\prime} \longmapsto \mathrm{u}^{\prime}$ with $\mathrm{u}^{\prime}: \mathrm{X} \longrightarrow \mathrm{K}$ where $\eta, \lambda$ and $\kappa, \varepsilon$ are the unit-counit pairs for the adjunctions $f \longmapsto u$ and those for $\mathrm{f}^{\prime} \longmapsto \mathrm{u}^{\prime}$ are similar except that they have primes. The counit for $\mathrm{u}^{\prime} \mathrm{u} \dashv \mathrm{ff}^{\prime}$ is

$$
\mathrm{u}^{\prime} \mathrm{uff} \stackrel{\mathrm{u}^{\prime} \varepsilon \mathrm{f}^{\prime}}{\longrightarrow} \mathrm{u}^{\prime} \mathrm{f}^{\prime} \stackrel{\varepsilon^{\prime}}{\longrightarrow} 1_{\mathrm{K}}
$$

while the unit for $f f^{\prime} \dashv u^{\prime} u$ is

$$
1_{K} \stackrel{\eta^{\prime}}{\longrightarrow} u^{\prime} f^{\prime} \stackrel{u^{\prime} \eta f^{\prime}}{\longrightarrow} u^{\prime} u f f^{\prime} .
$$

But $\varepsilon \circ \eta=\omega_{X}$ and $\varepsilon^{\prime} \circ \eta^{\prime}=\omega_{\mathrm{K}}^{\prime}$. So

$$
\begin{aligned}
\varepsilon^{\prime} \circ\left(\mathrm{u}^{\prime} \varepsilon \mathrm{f}^{\prime}\right) \circ\left(\mathrm{u}^{\prime} \eta \mathrm{f}^{\prime}\right) \circ \eta^{\prime} & =\varepsilon^{\prime} \circ\left(\mathrm{u}^{\prime}(\varepsilon \circ \eta) \mathrm{f}^{\prime}\right) \circ \eta^{\prime}=\varepsilon^{\prime} \circ\left(\mathrm{u}^{\prime} \omega_{\mathrm{X}} \mathrm{f}^{\prime}\right) \circ \eta^{\prime}=\varepsilon^{\prime} \circ\left(\omega_{\mathrm{K}} \mathrm{u}^{\prime} \mathrm{f}^{\prime}\right) \circ \eta^{\prime} \\
& =\varepsilon^{\prime} \circ \eta^{\prime} \circ \omega_{\mathrm{K}}=\omega_{\mathrm{K}}^{\prime} \circ \omega_{\mathrm{K}}=\left(\omega^{\prime} \circ \omega\right)_{\mathrm{K}} .
\end{aligned}
$$

A similar argument applies for the other composite. QED

Definition 4.2 A Frobenius monad $t=(t, \eta, \mu, \varepsilon, \delta)$ on an object $X$ of $\mathcal{D}$ is strongly separable when there exist invertible scalars $\omega$ and $\varpi$ such that the composites

$$
1_{X} \stackrel{\eta}{\longrightarrow} \mathrm{t} \stackrel{\varepsilon}{\longrightarrow} 1_{X} \text { and } \mathrm{t} \stackrel{\delta}{\longrightarrow} \mathrm{t} t \stackrel{\mu}{\longrightarrow} \mathrm{t}
$$

are equal to $\omega_{X}$ and $\bar{\omega}_{X} t$, respectively,

Proposition 4.2 A morphism in $\mathcal{D}$ is a projective equivalence if and only if it is monadic via a strongly separable Frobenius monad.

Proof A projective equivalence $\mathrm{u}: \mathrm{A} \longrightarrow \mathrm{X}$ is conservative since the counit $\lambda$ for its left adjoint is a retraction (split epimorphism). Since $\mathrm{u}$ also has a right adjoint, it is monadic. The composites $\varepsilon \circ \eta$ and $\mu \circ \delta=\mathrm{u} \lambda \mathrm{f} \circ \mathrm{u} \kappa \mathrm{f}=\mathrm{u}(\lambda \circ \kappa) \mathrm{f}$ are invertible scalars from Definition 4.1. So $\mathrm{u}$ generates a strongly separable Frobenius monad on $\mathrm{X}$.

Conversely, suppose we have a strongly separable Frobenius monad $\mathbf{t}=(\mathrm{t}, \eta, \mu, \varepsilon, \delta)$ on $\mathrm{X}$ and $\mathrm{u}: \mathrm{A} \longrightarrow \mathrm{X}$ together with action $\xi: \mathrm{t} u \longrightarrow \mathrm{u}$ provide an Eilenberg-Moore construction for $\mathbf{t}$. So there exists $\mathrm{f} \longmapsto \mathrm{u}$ where $\mathrm{uf}$ is isomorphic to $\mathrm{t}$ and $\xi \mathrm{f}$ transports to $\mu$. We can replace $\mathrm{t}$ by $\mathrm{uf}$ so that $\xi \mathrm{f}=\mu$. Then $\eta$ is the unit for $\mathrm{f} \dashv \mathrm{u}$ and $\varepsilon$ is the 
counit for $\mathrm{u} \dashv \mathrm{f}$. So we have that $\varepsilon \circ \eta$ is an invertible scalar. The counit $\kappa$ for $\mathrm{f} \dashv \mathrm{u}$ is determined by $\mathrm{u} \kappa=\xi$ and unit $\lambda$ for $\mathrm{u} \dashv \mathrm{f}$ is determined by

$$
\mathrm{u} \lambda=\mathrm{t} \xi \circ \delta \mathrm{u} \circ \eta \mathrm{u} \text {. }
$$

So

$$
\mathrm{u}(\kappa \circ \lambda)=\xi \circ \mathrm{t} \xi \circ \delta \mathrm{u} \circ \eta \mathrm{u}=\xi \circ \mu \mathrm{u} \circ \delta \mathrm{u} \circ \eta \mathrm{u}=\xi \circ \bar{\varpi}_{X} \mathrm{u} \circ \eta \mathrm{u}=\bar{\varpi}_{X} \mathrm{u} \circ \xi \circ \eta \mathrm{u}=\bar{\varpi}_{X} \mathrm{u}=\mathrm{u} \bar{\varpi}_{\mathrm{A}},
$$

which implies that $\kappa \circ \lambda=\varpi_{\mathrm{A}}$. Therefore $\mathrm{u}$ is a projective equivalence. QED

\section{§5. Variations on Morita equivalence}

Suppose $\mathcal{A}$ is a monoidal $\mathcal{V}$-category (that is, a pseudomonoid in $\mathcal{V}$-Cat). Then $\mathcal{P} \mathcal{A}$ becomes a cocomplete monoidal $\mathcal{V}$-category via the convolution tensor product

$$
(\mathrm{M} * \mathrm{~N})(\mathrm{A})=\int^{\mathrm{X}, \mathrm{Y}} \mathcal{A}(\mathrm{A}, \mathrm{X} \otimes \mathrm{Y}) \otimes \mathrm{MX} \otimes \mathrm{NY}
$$

of Brian Day [Dy]. Monoidal $\mathcal{V}$-categories $\mathcal{A}$ and $\mathcal{B}$ are defined to be Cauchy equivalent when $\mathscr{P A}$ and $\mathscr{P B}$ are equivalent monoidal $\mathcal{V}$-categories (that is, equivalent in the 2-category of monoidal $\mathcal{V}$-categories and monoidal $\mathcal{V}$-functors). Scott Johnson [Jn1] showed that the convolution tensor product on $\mathcal{P} \mathcal{A}$ restricts to $Q \mathcal{A}$ and that monoidal $\mathcal{V}$-categories $\mathcal{A}$ and $\mathcal{B}$ are Cauchy equivalent if and only if $\mathcal{Q A}$ and $Q \mathcal{B}$ are equivalent monoidal $\mathcal{V}$-categories. Moreover $\mathcal{A}$ is Cauchy equivalent to $Q \mathcal{A}$ as monoidal $\mathcal{V}$-categories.

From this we see in particular that Cauchy equivalence, monoidal or not, is a special case of equivalence once we find the appropriate ambient bicategory. Having in Section 3 weakened the notion of equivalence to projective equivalence, we can now contemplate projective Cauchy equivalence. Two $\mathcal{V}$-categories $\mathcal{A}$ and $\mathcal{B}$ are projectively Cauchy equivalent when $\mathcal{P} \mathcal{A}$ and $\mathcal{P B}$ are projectively equivalent $\mathcal{V}$-categories. For $\mathcal{V}$ equal to the category of sets or the category of abelian groups, this concept is the same as Cauchy equivalence. However, if $\mathcal{V}$ is the category of vector spaces over a field, for example, we do obtain a weaker kind of equivalence; in particular, this applies to associative unital algebras over the field. In general, $\mathcal{A}$ and $\mathcal{B}$ are projectively Cauchy equivalent if and only if $Q \mathcal{A}$ and $Q \mathcal{B}$ are projectively equivalent $\mathcal{V}$-categories.

Similarly, two monoidal $\mathcal{V}$-categories $\mathcal{A}$ and $\mathcal{B}$ are projectively Cauchy equivalent when $\mathscr{P A}$ and $\mathcal{P B}$ are projectively equivalent monoidal $\mathcal{V}$-categories. If $\mathcal{V}$ is the category of vector spaces over a field k, we do obtain a weaker kind of Cauchy equivalence for monoidal klinear categories. In general, monoidal $\mathcal{V}$-categories $\mathcal{A}$ and $\mathcal{B}$ are projectively Cauchy equivalent if and only if $Q \mathcal{A}$ and $Q \mathcal{B}$ are projectively equivalent monoidal $\mathcal{V}$-categories.

\section{§6. Wreath products of Frobenius algebras}

As in Section 1, we express our results in terms of monads rather than algebras.

We shall begin by recalling some notions from [LSt]. Given a 2-category $\mathcal{K}$ there is a 2- 
category $\operatorname{EM}(\mathcal{K})$ which turns out to be the free completion of $\mathcal{K}$ with respect to the Eilenberg-Moore construction on monads. Explicitly, an object of $\operatorname{EM}(\mathcal{K})$ is a pair $(\mathrm{A}, \mathrm{t})$ where $\mathrm{A}$ is an object of $\mathcal{K}$ and $\mathrm{t}$ is a monad on $\mathrm{A}$ in $\mathcal{K}$. A morphism $(\mathrm{f}, \phi):(\mathrm{A}, \mathrm{t}) \longrightarrow(\mathrm{B}, \mathrm{s})$ consists of a morphism $\mathrm{f}: \mathrm{A} \longrightarrow \mathrm{B}$ and 2-cell $\phi: \mathrm{s} \circ \mathrm{f} \Rightarrow \mathrm{f} \circ \mathrm{t}$ in $\mathcal{K}$ satisfying two compatibility conditions with the units $\eta$ and multiplications $\mu$ of the monads $\mathrm{t}$ and s. A 2-cell $\rho:(\mathrm{f}, \phi) \Rightarrow(\mathrm{g}, \psi)$ is a 2-cell $\rho: \mathrm{f} \Rightarrow \mathrm{g} \circ \mathrm{t}$ in $\mathcal{K}$ such that

$$
(\mathrm{g} \circ \mu)(\rho \circ \mathrm{t}) \phi=(\mathrm{g} \circ \mu)(\psi \circ \mathrm{t})(\mathrm{s} \circ \rho) \text {. }
$$

Composition in the category $\operatorname{EM}(\mathcal{K})((\mathrm{A}, \mathrm{t}),(\mathrm{B}, \mathrm{s}))$ involves using the multiplication $\mu$ of $\mathrm{t}$. Horizontal composition is straightforward.

A wreath in $\mathcal{K}$ is defined in [LSt] to be a monad in $\operatorname{EM}(\mathcal{K})$. Explicitly, a wreath consists of an object $\mathrm{A}$ of $\mathcal{K}$, monad $\mathrm{t}$ on $\mathrm{A}$, a morphism $\mathrm{s}: \mathrm{A} \longrightarrow \mathrm{A}$, and 2-cells $\lambda: \mathrm{t} \circ \mathrm{s} \Rightarrow \mathrm{s} \circ \mathrm{t}$, $\mathrm{l}: 1_{\mathrm{A}} \Rightarrow \mathrm{s} \circ \mathrm{t}$ and $\mathrm{v}: \mathrm{s} \circ \mathrm{s} \Rightarrow \mathrm{s} \circ \mathrm{t}$ satisfying seven conditions (on top of those that say $\mathrm{t}$ is a monad). Notice that $s$ need not itself be a monad, but it could be while $r$ and $v$ could be obtained from the unit and multiplication: in this case $\lambda$ is called a distributive law between the monads $\mathrm{t}$ and $\mathrm{s}$.

For any wreath, we obtain a monad structure on the composite endomorphism $\mathrm{s} \circ \mathrm{t}$. The unit is $\mathbf{l}$ and the multiplication is the composite

$$
\mathrm{s} \circ \mathrm{t} \circ \mathrm{s} \circ \mathrm{t} \stackrel{\mathrm{s} \circ \lambda \circ \mathrm{t}}{\longrightarrow} \mathrm{s} \circ \mathrm{s} \circ \mathrm{t} \circ \mathrm{t} \stackrel{v \circ \mu}{\longrightarrow} \mathrm{s} \circ \mathrm{t} \circ \mathrm{t} \stackrel{\mathrm{s} \circ \mu}{\longrightarrow} \mathrm{s} \circ \mathrm{t} \text {. }
$$

This composite monad is the wreath product.

A wreath is called Frobenius when the monad $(\mathrm{s}, \lambda)$ on $(\mathrm{A}, \mathrm{t})$ in $\operatorname{EM}(\mathcal{K})$ is equipped with a Frobenius structure. We leave this to the reader to make more explicit.

Proposition 6.1 The wreath product of a Frobenius wreath on a Frobenius monad $(\mathrm{A}, \mathrm{t})$ is Frobenius.

Proof Without loss of generality we may suppose $\mathcal{K}$ admits the Eilenberg-Moore construction. We use Theorem $1.6(\mathrm{f})$. Since $(\mathrm{A}, \mathrm{t})$ is Frobenius, the left adjoint $\mathrm{f}^{\mathrm{t}}$ to $\mathrm{u}^{\mathrm{t}}: \mathrm{A}^{\mathrm{t}} \longrightarrow \mathrm{A}$ is also right adjoint. The Eilenberg-Moore construction on the wreath, as a monad in $\operatorname{EM}(\mathcal{K})$ is the wreath product. So the left adjoint of the underlying $(\mathrm{A}, \mathrm{S} \circ \mathrm{t}) \longrightarrow(\mathrm{A}, \mathrm{t})$ is also a right adjoint. Since all 2-functors preserve adjunctions, the 2functor $\operatorname{EM}(\mathcal{K}) \longrightarrow \mathcal{K}$ assigning to each monad its Eilenberg-Moore construction, assigns to $(\mathrm{A}, \mathrm{S} \circ \mathrm{t}) \longrightarrow(\mathrm{A}, \mathrm{t})$, a morphism $\mathrm{A}^{\mathrm{s}} \longrightarrow \mathrm{A}^{\mathrm{t}}$ whose left adjoint is also its right adjoint. The 
underlying $\mathrm{A}^{\text {sot }} \longrightarrow \mathrm{A}$ is the composite of the underlyings $\mathrm{A}^{\text {sot }}$ $\mathrm{A}^{\mathrm{t}}$ A, and so has a two-sided adjoint. It follows then that $\mathrm{s} \circ \mathrm{t}$ is Frobenius. QED

\section{References}

[Bz] John Baez <http://math.ucr.edu/home/baez/>.

[Ba1] M. Barr, *-Autonomous categories, with an appendix by Po Hsiang Chu. Lecture Notes in Mathematics 752 (Springer, Berlin, 1979).

[Ba2] M. Barr, Nonsymmetric *-autonomous categories, Theoretical Computer Science 139 (1995) 115-130.

[Ba3] M. Barr, The Chu construction, Theory Appl. Categories 2 (1996) 17-35.

[BW1] J.W. Barrett and B.W. Westbury, Invariants of piecewise-linear 3-manifolds, Transactions Amer. Math. Soc. 348 (1996) 3997-4022.

[BW2] J.W. Barrett and B.W. Westbury, Spherical categories, Advances in Math. 143 (1999) 357-375.

[Bec] Jonathon Beck, Triples, algebras and cohomology, Repr. Theory Appl. Categ. No. 2(2003) 1-59.

[Bu] J. Bénabou, Introduction to bicategories, Lecture Notes in Math. 47 (Springer, Berlin, 1967) 1- 77.

[BS] Julien Bichon and Ross Street, Militaru's D-equation in monoidal categories, Applied Categorical Structures 11 (2003) 337-357.

[BJ] Stephen Boyer and André Joyal, Separable algebras and Seifert surfaces, Preprint (1994).

[Cbn] Aurelio Carboni, Matrices, relations and group representations, J. Algebra 136 (1991) 497-529.

[CW] Aurelio Carboni and Robert Walters, Cartesian bicategories I, J. Pure Appl. Algebra 49 (1987) 11-32.

[Cmd] Sean Carmody, "Cobordism Categories" (PhD Thesis, University of Cambridge, August 1995).

[Dy] B.J. Day, On closed categories of functors, Lecture Notes in Math.137 (Springer, Berlin, 1970) 1- 38.

[DMS] Brian Day, Paddy McCrudden and Ross Street, Dualizations and antipodes, Applied Categorical Structures 11 (2003) 229-260.

[DS1] Brian Day and Ross Street, Monoidal bicategories and Hopf algebroids, Advances in Math. 129 (1997) 99-157.

[DS2] Brian Day and Ross Street, Quantum categories, star autonomy, and quantum groupoids, in "Galois Theory, Hopf Algebras, and Semiabelian Categories", Fields Institute Communications 43 (American Math. Soc. 2004) 193-231.

[Dd] V.G. Drinfel'd, Quasi-Hopf algebras (Russian), Algebra i Analiz 1 \#6 (1989) 114-148; translation in Leningrad Math. J. 1 \#6 (1990) 1419-1457.

[EM] Samuel Eilenberg and John C. Moore, Adjoint functors and triples, Illinois J. Math.9(1965) 381-398.

[Frd] P.J. Freyd, P.W. O'Hearn, A.J. Power, R. Street, M. Takeyama, and R.D. Tennent, Bireflectivity, Theoretical Computer Science 228 (1-2) (1999) 49-76.

[Jn1] S.R. Johnson, Monoidal Morita equivalence, J. Pure Appl. Algebra 59 (1989) 169-177.

[Jn2] S.R. Johnson, Small Cauchy completions, Journal of Pure \& Appl. Algebra 62 (1989) 35-45.

[JS1] André Joyal and Ross Street, The geometry of tensor calculus I, Advances in Math. 88 (1991) 55-112.

[JS2] André Joyal and Ross Street, Tortile Yang-Baxter operators in tensor categories, J. Pure Appl. Algebra 71 (1991) 43-51. 
[JS3] André Joyal and Ross Street, An introduction to Tannaka duality and quantum groups, Lecture Notes in Math. 1488 (Springer-Verlag Berlin, Heidelberg, New York 1991) 411-492.

[Kas] Christian Kassel, Quantum Groups, Graduate Texts in Math. 155 (Springer-Verlag Berlin, Heidelberg, New York 1995).

[Kel] G.M. Kelly, Basic Concepts of Enriched Category Theory, London Math. Soc. Lecture Notes Series 64 (Cambridge University Press 1982).

[KS] G.M. Kelly and Ross Street, Review of the elements of 2-categories, Lecture Notes in Math. 420 (1974) 75-103.

[KL] Thomas Kerler and Volodymyr L. Lyubashenko, Non-Semisimple Topological Quantum Field Theories for 3-Manifolds with Corners, Lecture Notes in Mathematics 1765 (Springer, Berlin, 2001).

[Ko] Joachim Kock, Frobenius Algebras and 2D Topological Quantum Field Theories, London Math. Soc. Student Texts 59 (Cambridge University Press, 2003).

[LSt] Stephen Lack and Ross Street, The formal theory of monads II, J. Pure Appl. Algebra 175 (2002) 243-265.

[LSw] R.G. Larson and M.E. Sweedler, An associative orthogonal bilinear form for Hopf algebras, Amer. J. Math. 91 (1969) 75-94.

[Law1] F.W. Lawvere, Ordinal sums and equational doctrines, in: "Seminar on Triples and Categorical Homology Theory", Lecture Notes in Math. 80 (1969) 141-155.

[Law2] F.W. Lawvere, Metric spaces, generalized logic, and closed categories, Rendiconti Semi. Mat. Fis. Milano 43 (1974) 135-166.

[ML] S. Mac Lane, Categories for the Working Mathematician, Graduate Texts in Math. 5 (SpringerVerlag, 1971).

[Maj] Shahn Majid, Foundations of Quantum Group Theory (Cambridge University Press, 1995).

[McC] Paddy McCrudden, Categories of Representations of Balanced Coalgebroids (PhD Thesis, Macquarie University, 22 February 1999).

[Mü1] Michael Müger, From subfactors to categories and topology. I: Frobenius algebras in and Morita equivalence of tensor categories, J. Pure Appl. Algebra 180 (2003) 81-157.

[Mü2] Michael Müger, From subfactors to categories and topology. II: The quantum double of tensor categories and subfactors, J. Pure Appl. Algebra 180 (2003) 159-219.

[St1] Ross Street, The formal theory of monads, J. Pure Appl. Algebra 2 (1972) 149-168.

[St2] Ross Street, Enriched categories and cohomology, Quaestiones Math. 6 (1983) 265-283.

[St3] Ross Street, Absolute colimits in enriched categories, Cahiers topologie et géométrie différentielle 24 (1983) 377- 379.

[St4] Ross Street, Higher categories, strings, cubes and simplex equations, Applied Categorical Structures 3 (1995) 29- 77 \& 303.

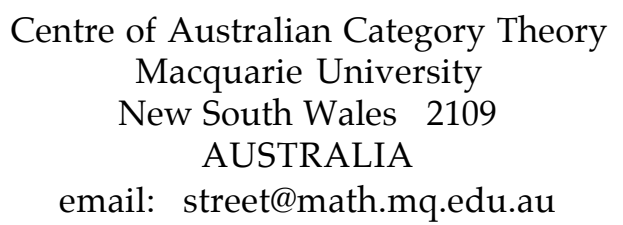

